

\section{DISCLAIMER}

This report was prepared as an account of work sponsored by an agency of the United States Government. Neither the United States Government nor any agency Thereof, nor any of their employees, makes any warranty, express or implied, or assumes any legal liability or responsibility for the accuracy, completeness, or usefulness of any information, apparatus, product, or process disclosed, or represents that its use would not infringe privately owned rights. Reference herein to any specific commercial product, process, or service by trade name, trademark, manufacturer, or otherwise does not necessarily constitute or imply its endorsement, recommendation, or favoring by the United States Government or any agency thereof. The views and opinions of authors expressed herein do not necessarily state or reflect those of the United States Government or any agency thereof. 


\section{DISCLAIMER}

Portions of this document may be illegible in electronic image products. Images are produced from the best available original document. 


\section{Printed in the United States of America. Available from National Technical Information Service \\ U.S. Department of Commerce 5285 Port Royal Road, Springfield, Virginia 22161 NTIS price codes-Printed Copy: A04; Microfiche A01}

This report was prepared as an account of work sponsored by an agency of the United States Government. Neither the United States Government nor any agency thereof, nor any of their employees, makes any warranty, express or implied, or assumes any legal liability or responsibility for the accuracy, completeness, or usefulness of any information, apparatus, product, or process disclosed, or represents that its use would not infringe privately owned rights. Reference herein to any specific commercial product, process, or service by trade name, trademark, manufacturer, or otherwise, does not necessarily constitute or imply its endorsement, recommendation, or favoring by the United States Government or any agensy thereof. The views and opinions of authors expressed herein do not necessarily state or reflect those of the United States Government or any agency thereof. 
ORNL/TM-7132

Dist. Category UC- $20 \mathrm{~g}$

\author{
Contract No. N-7405-eng-26 \\ FUSION ENERGY DIVISION
}

\title{
3-D NONLINEAR CALCULATIONS OF RESISTIVE TEARING MODES
}

\author{
H. R. Hicks \\ J. A. Holmes \\ D. K. Lee \\ Computer Sciences Division \\ and \\ B. Carreras \\ B. V. Wadde $11^{*}$ \\ Fusion Energy Division
}

NOTICE This document contains information of a preliminary nature. It is subject to revision or correction and therefore does not represent a final report.

Date Published - March 1981

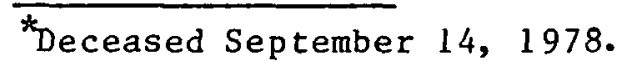

Prepared by the

OAK RIDGE NATIONAL LABORATORY

Oak Ridge, Tennessee 37830

operated by

UNION CARBIDE CORPORATION

for the

DEPARTMENT OF ENERGY

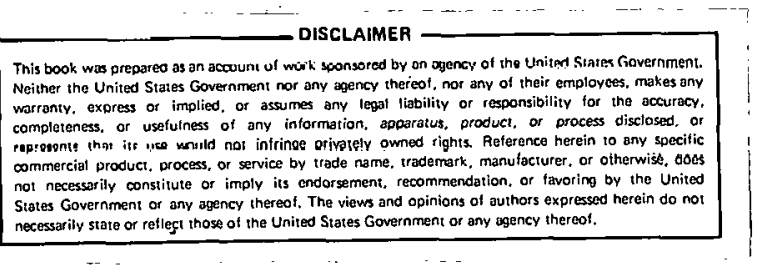

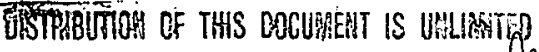


THIS PAGE

\section{WAS INTENTIONALLY LEFT BLANK}


CONTENTS

ABSTRACT . . . . . . . . . . . . . . . . . . . . . 1

1. INTRODUCTION . . . . . . . . . . . . . . . . . . . 3

2. THE EQUATIONS . . . . . . . . . . . . . . . . . . . . . . 7

2.1 INITIAL CONDITIONS . . . . . . . . . . . . . . . 10

2.2 BOUNDARY CONDITTONS . . . . . . . . . . . . . . . 13

2.3 ENERGY CONSERVATION . . . . . . . . . . . . . . . 14

3. NUMERICAL SCHEMES . . . . . . . . . . . . . . . . . 17

3.1 MASS ...................... . 17

3.2 RS3 . . . . . . . . . . . . . . . . . 19

3.3 RSF . . . . . . . . . . . . . . . . . . 21

3.3.1 Equations . . . . . . . . . . . . . . 22

3.3.2 Choice of Modes . . . . . . . . . . . 25

3.3.3 Convolution . . . . . . . . . . . . . . 28

3.3.4 Timestep . . . . . . . . . . . . . . . . . . 30

3.3.5 Grid . . . . . . . . . . . . . . 32

3.3.6 Sources of Numerical Error . . . . . . . . 32

3.3.7 Output . . . . . . . . . . . . . . 34

4. COMPARISON OF CODES . . . . . . . . . . . . . . . . . . . 39

REFERENCES . . . . . . . . . . . . . . . . . . . . . . 41 


\begin{abstract}
Recent numerical calculations of the evolution of resistive tearing modes have been central to the understanding of magnetohydrodynamic activity and disruptions in tokamaks. The nonlinear, 3-D, inftial-value computer code RSF has provided many of these results. Th1s code assumes cylindrical geometry with a Fourler series representation in the two periodic coordinates and a finite-difference representation in the radial direction. This choice makes RSF considerably more accurate and efficient than previous codes.
\end{abstract}


THIS PAGE

\section{WAS INTENTIONALLY LEFT BLANK}




\section{INTRODUCTION}

The tokamak has emerged as the most promising magnetic confinement device for achieving controlled nuclear fusion. Tokamak plasmas exhibit a variety of large-scale oscillations and instabilities. ${ }^{1}$ These range from those that limit the plasma density or confinement time to major disruptions that violently terminate the discharge and possibly damage the device. It is important for the design and operation of future devices to understand these plasma instabilities, especially the major disruptions. Over the last few years, resistive magnetohydrodynamic (MHD) calculations have produced a wealth of results ${ }^{2-17}$ that have contributed substantially to the understanding of many of these features of tokamak discharges. It has even been possible to suggest ways of controlling some of the most serious plasma disruptions. 13

The resistive MHD equations, when linearized about an equilibrium, exhibit exponential solutions ${ }^{18}$ that are driven by the gradient of the toroldal current density. They are called tearing modes because the flinx-surface topology is torn. However, nonlinear effects become important before the linear solutions are large enough to be experimentally measurable. The nonlinear evolution can be studied by solving the resistive MHD equations as an inftial-value problem. If the initial condition is that of an unstable equilibrium plus a small perturbation, then the time integration of the partial differential equations (PDEs) will first exhibit the growth rates and eigenfunctions of the fastest growing solutions of the Linearized equations. If several modes are unstable and if they have different pitches, then a 
3-D calculation is required for the nonlinear phase. Calculations of this type have yielded results that are qualitatively distinct from the 2-D results.

It is necessary to follow phenomena simultaneously on two time scales: ' $\mathrm{Hp}$ ' the transit time of an Alfven wave in the poloidal direction; and ' $r$ ' the resistive diffusion time of the plasma. Unfortunately, in present-day tokamaks the ratio of these times is very large,

$$
\frac{\tau_{r}}{\tau_{H p}} \equiv S \sim 10^{5}-10^{8} \text {, }
$$

and it may be even larger for reactor-sized devices.

In order to understand and verify these calculations, it is necessary to clearly separate $\tau_{H p}, \tau_{\underline{r}}$, and the intermediate time scale characteristic of the tearing mode growth rate. $\left(S^{1 / 3} \tau_{r}\right.$ or $S^{2 / 5} \tau_{r}$, depending on the symmetry of the solution). This leads to the requirement that $S$ to the fractional power be quite large in order to distinguish the phases and time scales of the problem in a numerical calculation. For example, it is known ${ }^{1 y}$ that the exponential growth phase is followed by a nonlinear phase characterized by slower algebraic growth. It is important to show that, in certain cases, faster growth can return after a well-established period of algebraic growth. This is not possible for $S \leqslant 10^{5}$ because the algebraic growth does not have sufficient time to become clearly established. In the later phases of a disruption at high $S$, the plasma can enter a state that might be regarded as turbulent. The level of turbulence decreases 
as $\mathrm{S}$ is reduced. For $\mathrm{S} \leqslant 10^{4}$, some modes that are unstable at high $\mathrm{S}$ do become stable. This can imply a critical value of $\mathrm{S}$, below which turbulence is absent. Also, at low $S$ the $p=1$ helicity (described later) saturates, while at high $\mathrm{S}$ it reconnects. For these reasons, we conclude that low S calculations are inappropriate for understanding phenomena that occur at $s \sim 10^{6}$. On the other hand, for $S \rightarrow \infty$, the equations become singular. ${ }^{18}$ Therefore, at high values of $S$ the spatial structure of the solutions becomes quite small and requires a numerical scheme capable of resolving short wavelengths in the minor radius direction. These requirements of disparate time scales and fine radial mesh imply that such calculations are time-consuming even on the fastest computers available today. Consequently, execution efficiency is an important issue for any such code.

This paper primarily describes the code RSF, which was designed with the above conditions in mind. It uses a Fourier series expansion in two periodic coordinates and a finite-difference grid in the third. Its performance is compared with the less efficient and less accurate 3-D code RS3 $3^{10}$ and with an earlier 2-D code MASS. ${ }^{6}$ Both codes use a finite-difference technique. Results obtained with RSF have been extensively reported elsewhere, ${ }^{1-15}$ so the ones given here are used merely to illustrate the capabilities of the code.

In Sect. 2 the equations and initial and boundary conditions are described. The numerical schemes of MASS and RS3 are briefly described in Sects. 3.1 and 3.2. Then in Sect. 3.3 a detailed description of RSF is givel. In Sect. 4, a comparison of these codes is made. 
6

Blank 
2. THE EQUATIONS

The full resistive MHD equations ${ }^{10}$ (Ampere's law, Faraday's law, momentum balance, $\mathrm{Ohm}^{\prime} \mathrm{s}$ law, equation of state, mass continuity, and resistivity evolution) can be considerably simplified ${ }^{10,20}$ by the application of two assumptions.

The first assumption is standard tokamak (inverse aspect ratio) ordering. That is,

$$
\frac{\mathrm{B}_{\theta}}{\mathrm{B}_{\zeta}} \sim \varepsilon,
$$

where

$$
\varepsilon \equiv \frac{a}{R_{0}} \ll 1
$$

Here $a$ is the minor radius and $R_{0}$ is the major radius of the torus. A cylindrical coordinate system $(r, \vartheta, \zeta)$ is employed, where $r(0 \leqslant r \leqslant a)$ is the minor radius, $\theta(0 \leqslant \theta \leqslant 2 \pi)$ is the poloidal angle, and $\zeta(0 \leqslant \zeta \leqslant 2 \pi)$ is the toroidal (or longitudinal) angle. In the circular cylinder limit, only terms of order $\varepsilon^{0}$ are retained and the cross-sectional shape of the plasma is assumed circular. This ordering has the important effect of allowing the time variation of the toroidal magnetic field ${ }_{\zeta}$ to be ignored; consequently, the fastest time scale of the equations, the time for the propagation of Alfven waves across the magnetic field, is removed from the dynamics. The fastest time scale remaining in the equations is $\tau_{H p}=R_{o} / V_{A}$, the time for Alfven 
waves to propagate along the magnetic field. The slow time scale is the resistive skin time $\tau_{r} \equiv a^{2} \mu_{0} / \bar{n}$.

The second assumption involves consideration of a low- $\beta$ plasma only ( $\beta$ is the ratio of plasma pressure to magnetic field pressure). Specifically, $B \sim \varepsilon^{2}$ is assumed. This is valid for most ohmically heated tokamaks.

This deliberate strategy of aiming for efficient, nonlinear, $3-\bar{D}$, high S calculations while dropping toroldal, noncircular, and finite- B effects has borne, fruit. Even though the equations are greatly simplified, they do contain the essential physics. The calculations have yielded quantitative agreement with a wide range of experimental observations.

The above assumptions yield two scalar PDEs that, in dimensionless form, are ${ }^{10}$

$$
\frac{\mathrm{D} \Psi}{\mathrm{Dt}}=11 \mathrm{~J}_{\zeta}-\frac{\partial \Phi}{\partial \zeta}-\mathrm{E}_{\zeta}^{\mathrm{W}}
$$

and

$$
\frac{D I I}{D t}=-s^{2}\left[\hat{\zeta} \cdot\left(\vec{V} \Psi \times \vec{\nabla} J_{\zeta}\right)+\frac{\partial J_{\zeta}}{\partial \zeta}\right]
$$

where

$\Psi \quad$ is the poloidal flux function (normalized to $\mathrm{a}^{2} \mathrm{~B}_{\zeta O}$ ) defined by $\vec{B}=(-\varepsilon \vec{\nabla} \Psi \times \hat{\zeta}+\hat{\zeta}) B_{\zeta O}$,

$\underset{\mathrm{Dt}}{\mathrm{D}} \quad$ is the convective derivative $\frac{\partial}{\partial \mathrm{t}}+\overrightarrow{\mathrm{v}}_{\perp} \cdot \vec{\nabla}$,

$\vec{v}$ is the fluid velocity in units of $a / \tau_{r}$,

$\perp$ denotes perpendicularity to $\hat{\zeta}$,

$n$ is the resistivity normalized to unity at the magnetic axis, 


$$
\begin{aligned}
& \mathrm{J}_{\zeta}=\nabla_{\perp}^{2} \Psi \text { is the toroidal component of the } \\
& \text { plasma current density normalized to } \mu_{0} \mathrm{R}_{0} / \mathrm{B}_{\zeta \circ}, \\
& \Phi \quad \text { is the velocity stream function, } \vec{v}_{\perp}=\vec{\nabla}_{\Phi} \times \hat{\zeta}, \\
& \mathrm{E}_{\zeta} \text { is the equilibrium toroidal electric field at the wall, and } \\
& \mathrm{U} \equiv \nabla_{\perp}^{2} \text { is the toroidal vorticity. }
\end{aligned}
$$

Most of these field quantities $\left(\Psi, J_{\zeta}, \Phi, U, \vec{B}_{\perp}\right.$, and $\left.\vec{v}_{\perp}\right)$ are functions of $r, \theta, \zeta$, and $t$. Except in Ref. 15, where the electron heat conduction equation is used to determine $\eta$, it is assumed that

$$
n(r)=\frac{E_{\zeta}^{W}}{J_{\zeta o}(r)}
$$

This removes from the equations the resistive decay of the plasma. The subscript o denotes an equilibrium quantity, and the tilde $(\sim)$ will be used to denote the nonequilibrium (perturbed) portion of a field quantity; for example,

$$
\mathrm{J}_{\zeta} \equiv \mathrm{J}_{\zeta \mathrm{O}}+\tilde{\mathrm{J}}_{\zeta}
$$

These equations are in dimensionless form. Lengths have been normalized to the minor radius $a$, and the time has been normalized to ${ }^{\tau} r^{\cdot}$ 


\section{$2 \cdot 1$ INITIAL CONDITIONS}

The reduced equations [Eqs. (1) and (2)] have nontrivial, velocity-free equilibrium solutions, for which the field quantities depend only on $r$. Under these conditions, the right-hand side of Eq. (2) is zero, implying that the vorticity does not evolve. If the velocity is zero, then $\Phi_{0}=0$. Since $E_{\zeta}^{W}$ cancels with ${ }^{W J} J_{\zeta 0}$ by definition, the right-hand side of $\mathrm{Eq} \cdot(1)$ is also zero. Since this implies that $\Psi_{0}$ does not evolve, and consequently that $J_{\zeta_{0}}$ does not evolve, any such. solution is an equilibrium. Equations (1) and (2) become

$$
\frac{\partial}{\partial t} \Psi_{0}(r)=0
$$

and

$$
\Phi_{0}=U_{0}=0
$$

To specify an equilibrium, it is only necessary to specify the equilibrium poloidal flux $\Psi_{n}(r)$. However, it has instead been the practice to specify the safety factor $q(r)$ and then calculate the flux from

$$
\frac{d}{d r} \Psi_{0}(r)=-\frac{r}{q(r)}
$$

This approach is preferable because $q(r)$ gives the positions of the resonance surfaces. 
At least a dozen different parameterizations for $q(r)$ have been used, each one having from one to five independent parameters. These are useful for studying the systematic dependence of the evolution on the equilibrium. The most extensively used parameterization has been

$$
q(r)=q_{o}\left[1+\left(\frac{r}{r_{0}}\right)^{2 \lambda}\right]^{1 / \lambda},
$$

where $\lambda=\lambda_{0}+(1 / 2) \lambda_{0}^{\prime \prime} r^{2}$ and $q_{0}, r_{0}, \lambda_{0}$, and $\lambda_{0}^{\prime \prime}$ are input parameters. When profiles of the toroldal plasma current density or electron temperature are available from, for example, transport code analyses of experimental conditions, these can be used to establish the q profile using

$$
q(r) \propto \frac{r^{2}}{\int_{0}^{r} d r^{\prime} r^{\prime} J_{\zeta}\left(r^{\prime}\right)}
$$

or

$$
q(r) \propto \frac{r^{2}}{\int_{0}^{r} d r^{\prime} r^{\prime}\left[T_{e}\left(r^{\prime}\right)\right]^{3 / 2}} .
$$

In either case, the normalization is given by specifying $q$ at the plasma boundary, which is directly related to the total plasma current. The usual initial conditions are (a) zero for the velocity-related fields

$$
\Phi(r, \theta, \zeta, 0)=U(r, \theta, \zeta, 0)=0
$$


and (b) an equilibrium plus a small perturbation for the poloidal flux

$$
\Psi(r, \theta, \zeta, 0)=\Psi_{0}(r)+\tilde{\Psi}(r, \theta, \zeta),
$$

where $\Psi_{0}(r)$ is given by Eq. (4). To specify the perturbation, it is useful to expaud

$$
\ddot{\psi}(r, \theta, \zeta)=\sum_{m, n}\left[\ddot{\psi}_{m n}^{c}(r) \cos (m \theta+n \zeta)+\ddot{\psi}_{m n}^{s}(r) \sin (m \theta+n \zeta)\right]
$$

Note that the equilibrium is $(m=0 ; n=0)$ in character, so that $\Psi_{m n}^{s}=\tilde{\Psi}_{m n}^{s}$ and $\Psi_{m n}^{c}=\tilde{\Psi}_{m n}^{c}$, except for $\Psi_{o o}^{c}=\Psi_{o}+\tilde{\psi}_{o 0^{\circ}}^{c}$ The solutions of Eqs. (1) and (2) when linearized about an equilibrium are characterized by $m, n$ values. Thus, for an equilibrium that is unstable with respect to only the $(m=2 ; n=1)$ mode, the quickest way to approach the linear solution numerically is to initialize $\tilde{\Psi}_{21}^{c}(r)$ [or $\tilde{\Psi}_{21}^{s}(r)$ ] with a function that is qualitatively similar to the linear eigenfunction; for example,

$$
\tilde{\Psi}_{m n}^{c}(r)=-\left(\frac{W_{I}}{4}\right)^{2} \frac{r^{m}(1-r)}{r_{s}^{m}\left(1-r_{s}\right)} \frac{2}{1+e^{10\left(-1+r / r_{s}\right)}} \frac{r_{0} q^{\prime}}{q\left(r_{s}\right)^{2}},
$$

where $r_{s}$ is the resonant radius of the mode, given by

$$
q\left(r_{s}\right)=\frac{m}{n}
$$

and 


$$
\mathrm{q}^{\cdot}=\frac{\mathrm{dq}}{\mathrm{dr}} \mathrm{I}_{\mathrm{s}}
$$

The initial magnetic island width $\mathrm{W}_{\mathrm{I}}$ is used to control the size of the perturbation.

Although RSF allows both the sine and cosine terms, in cases where only the cosine terms in $\Psi$ are initialized, the sine terms in $\Psi$ and the cosine terms in $\Phi$ remain zero as time evolves. In order to simplify the presentation here, only this latter situation will be discussed; therefore the superscripts $c$ and $s$ will be dropped.

The numerical solutions are independent of the inital perturbation if the perturbation is sufficiently small. For a single helicity case, only one mode is normally perturbed. If the perturbation is sufficiently small that an eigenfunction emerges before the island width exceeds the tearing layer width, then the only role that the inftial perturbation amplitude plays in the solution is to determine the zero of time. In multihelicity runs, two modes of differing pitch are normally perturbed. The solution then depends on the relative magnitudes of the two perturbations in a way that is well understood. 15

\section{2 BOUNDARY CONDITIONS}

The field functions are assumed periodic in $\theta$ and $\zeta$. At the magnetic axis $(r=0)$, all scalar functions must be regular. Later, the implementation of this in the code RSF will be discussed.

The wall at $r=1$ is assumed rigid, so $v_{r}(1)=0$. This implies that $\Phi(1)$ is independent of $\theta$. Since only derivatives with respect to $\theta$ enter, we can choose 
$\Phi(1, \theta, \zeta, t)=0$

In Eqs. (1) and (2), $\Psi$ has been replaced by $\Psi-E_{\zeta}{ }^{t}$, giving either

$\Psi(1, \theta, \zeta, t)=0$

for constant voltage or

$$
\left.\frac{d}{d r} \int d \theta d \zeta \Psi(r, \theta, \zeta, t)\right|_{r=1}=\text { constant }
$$

for constant current boundary condition.

To study the effect of external feedback coils, ${ }^{13}$ it is necessary to modify the boundary condition of the mode subject to feedback,

$$
\mu_{m n}(1,0,5, t)-H_{\text {ext }}(0,5, t) \text {, }
$$

according to a prescription discussed in Ref. 13.

\subsection{ENERGY CONSERVATION}

The energy conservation law for the reduced MHD equations is obtained by multiplying $\mathrm{Eq} \cdot(1)$ by $-\mathrm{J}_{\zeta}$ and integrating over volume:

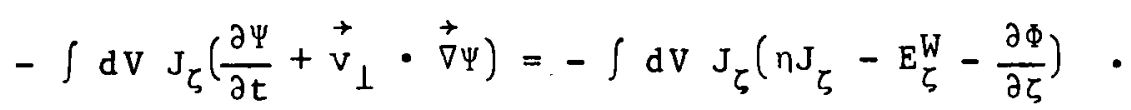

Integrating Eq. (14) by parts, applying the boundary condition on $\Phi$ [Eq. (12)], and using $\vec{\nabla} \cdot \vec{v}=0$ yields 


$$
\begin{gathered}
-\int \frac{\partial \Psi}{\partial t} \vec{\nabla}_{\perp} \Psi \cdot \vec{d} \vec{S}+\frac{d}{d t}\left[\frac{1}{2} \int\left(\vec{\nabla}_{\perp} \Psi\right)^{2} \cdot d V\right]+\int \vec{v}_{\perp} \Psi \cdot \vec{\nabla}_{\zeta} d V \\
=\int d V\left(-\eta J_{\zeta}^{2}+E_{\zeta}^{W} J_{\zeta}+\frac{\partial \Phi}{\partial \zeta} J_{\zeta}\right) .
\end{gathered}
$$

Similarly, multiplying Eq. (2) by $-\Phi / \mathrm{S}^{2}$ and integrating over volume gives

$$
-\frac{1}{\mathrm{~s}^{2}} \int \mathrm{dV} \Phi\left(\frac{\partial \mathrm{U}}{\partial \mathrm{t}}+\overrightarrow{\mathrm{v}}_{\perp} \cdot \vec{\nabla} \mathrm{U}\right)=\int \mathrm{dV} \Phi\left[\hat{\zeta} \cdot\left(\vec{\nabla} \Psi \times \vec{\nabla}_{\zeta}\right)+\frac{\partial}{\partial \zeta} \mathrm{J}_{\zeta}\right]
$$

or

$$
\frac{1}{s^{2}} \frac{d}{d t}\left[\frac{1}{2} \int d V\left(\vec{\nabla}_{\perp} \Phi\right)^{2}\right]=\int d v \vec{v}_{\perp}^{\Psi} \cdot \vec{\nabla}_{\zeta}+\int d v \frac{\partial J_{\zeta}}{\partial \zeta}
$$

Adding Eqs. (15) and (17),

$$
\begin{aligned}
& \frac{\mathrm{d}}{\mathrm{dt}}\left(\varepsilon_{\mathrm{M}}+\varepsilon_{\mathrm{K}}\right) \equiv \frac{\mathrm{d}}{\mathrm{dt}} \int \mathrm{dV}\left[\frac{1}{2}\left(\vec{\nabla}_{\perp} \Psi\right)^{2}+\frac{1}{2 \mathrm{~S}^{2}}\left(\vec{\nabla}_{\perp} \Phi\right)^{2}\right]=\int \frac{\partial \Psi}{\partial t} \vec{\nabla}_{\perp} \Psi \cdot \mathrm{dS} \\
& -\int \mathrm{dV} \eta J_{\zeta} \hat{J}_{\zeta} \equiv-F-Q,
\end{aligned}
$$

where $\varepsilon_{M}$ and $\varepsilon_{K}$ are magnetic and kinetic energy, respectively. Thus, the rate of change of the total plasma energy is equal to the sum of the flux through the wall and the Joule heating. 
16

Blank 
3. NUMERICAL SCHEMES

To integrate Eqs. (1) and (2) in time, it is necessary to start from a perturbed equilibrium. Three codes that will do this will be described in chronological order. The first two, MASS and RS3, have been described elsewhere; ${ }^{6,10}$ thus, only sufficient detail to allow comparison with RSF is given here.

\subsection{MASS}

The MASS code was developed by the joint effort of the resistive MHD groups in Princeton at PPPL and IAS. ${ }^{2,4,5}$ It was later modified and extensively used at ORNL. ${ }^{7,9}$ It is summarily described here only to give better perspective to RS3 and RSF.

The terms in Eq. (10) can be grouped by helicity. If $p \equiv \mathrm{m} / \mathrm{n}$, then the double sum can be restated as

$$
\tilde{\Psi}(r, \theta, \zeta, t)=\sum_{p} \sum_{n} \tilde{\psi}_{n p, n}(r, t) \cos [(p \theta+\zeta) n]
$$

where $p$ takes all rational values. If only perturbations of a single pitch (or helicity) $p$ are present, then, in cylindrical geometry where the equilibrium is $(m=0 ; n=0)$, other helicities are never generated.

The MASS code was developed to study these single helicity cases. It played a crucial role in analyzing both linear and nonlinear behavior of tearing modes. It was used to study the $p=1$ helicity as a model for sawtooth oscillations. ${ }^{7}$ This mode initially tears the flux-surface configuration but later restores it to nested flux contours through magnetic field line reconnection. 
Calculations for other helicities $(p \neq 1)$ show a period of exponential growth followed by saturation of the perturbation giving a new, stable, nonaxisymmetric final state. When $S$ is large $\left(\sim 10^{6}\right)$ there is a well-defined period prior to island saturation in which the perturbation slows from exponential growth to algebraic growth. This numerically supports the result of Rutherford. 19

The advantage of assuming only one value of $p$ in the right-hand side of Eq. (19) is that the coordinates $\theta$ and $\zeta$ enter only in the combination $o_{p} \equiv p \theta \mid \zeta$. Consequently, the piublell tall be reduced to two dimensions, $r$ and $\theta_{\mathbf{p}}$. The MASS code uses a finite-difference formulation on a 2-D polar grid. Because of symmetry, it is only necessary to employ a segment of the grid such that

$$
0 \leqslant \theta_{\mathrm{p}} \leqslant \frac{\pi}{\mathrm{m}_{\min }}
$$

However, getting answers that are even qualitatively good can require about 20 grid points in the $\theta_{p}$ direction (Figs. 1 and 2 ).

The MASS code cannot correctly calculate the evolution of equilibria that are strongly unstable to more than one helicity because it does not include any nonlinear effects between modes of unequal helicity. Since it was believed that some important new effects might be found here, a 3-D code called RS3 was developed. 


\subsection{RS3}

The generalization to three dimensions, which allows for the interaction of different helicities, is accomplished in RS3 by replacing the polar grid of the MASS code with a full cylindrical grid. In cases in which only one helicity is unstable or in which the unstable modes saturate at a sufficiently low level, RS3 duplicates the results of the MASS code. However, when more than one mode is unstable and when the single helicity saturation amplitudes are sufficiently large, the behavior departs from a superposition of single helicity solutions, and a qualitatively different solution is found.

From linear tearing mode theory, ${ }^{18}$ it is known that the resistivity plays a role only inside the tearing layer, which is a very narrow region surrounding the singular radius. The tearing layer width $\varepsilon_{T}$ is proportional to $\mathrm{s}^{-2 / 5}$. One expects, therefore, that a very fine radial grid is required, at least in the vicinity of the singular surfaces of unstable modes. In addition to this, the $\theta$ and $\zeta$ grids must be fine, not only to support a sufficient number of $m$ and $n$ values, but also to minimize a sensitive form of truncation error. Consider any function of the form

$$
f=\cos (m \theta+m \zeta)
$$

Finite-difference first derlvatives on an equally spaced grid are given by

$$
\frac{\delta f}{\delta \zeta}=-n\left[\frac{l-(n \Delta \zeta)^{2}}{6}\right] \sin (m \theta+n \zeta)+0\left[(\Delta \zeta)^{4}\right]
$$


and

$$
\frac{\delta \mathrm{f}}{\delta \theta}=-\mathrm{m}\left[\frac{1-(\mathrm{m} \Delta \theta)^{2}}{6}\right] \sin (\mathrm{m} \theta+\mathrm{n} \zeta)+0\left[(\Delta \theta)^{4}\right]
$$

The discretization errors,

$$
\frac{n_{\text {eff }}}{n}=\frac{1-(n \Delta \zeta)^{2}}{6}
$$

and

$$
\frac{m_{\text {eff }}}{m}=\frac{1-(m \Delta \theta)^{2}}{6}
$$

occur in any term with a $\zeta$ or $\theta$ derfvative. These factors reduce the contribution of the higher modes in such terms. The solutions are particularly sensitive to tertus of the form

$$
\frac{\delta \mathrm{f}}{\delta \zeta}-\frac{1}{\mathrm{q}} \frac{\delta \mathrm{f}}{\delta \theta}
$$

Such terms should go to zero at the resonant surface where $q=\mathrm{m} / \mathrm{n}$. This will be the case if the grid is chosen so that

$$
\frac{m_{\text {eff }}}{n_{\text {eff }}}=q \text {, }
$$

which implies that $\mathrm{n} \Delta \zeta=\mathrm{m} \Delta \theta$. Even the elimination of this part of the discretization error is only effective for a single helicity at a time. 
A $30 \times 15(\theta \times \zeta)$ grid gives the correct $\mathrm{m} / \mathrm{n}$ for the $2 / 1$ helicity, but there is a $5 \%$ error in the effective $\mathrm{m} / \mathrm{n}$ for a $3 / 2$ helicity on that grid. For a relatively flat q profile, this can considerably alter the radius of the resonant surface. In order to overcome these problems and the additional fact that RS3 is too slow to be useful in systematic studies, RSF was written.

\subsection{RSF}

To overcome the limitation in RS3 due to discretization in the $\theta$ and $\zeta$ directions, RSF is designed to use a Fourier series expansion in these two directions. Each of the field quantities is expanded as in Eq. (10), a finite set of terms is retained, and the amplitudes of each mode are discretized on an unequally spaced grid in minor radius:

$$
\mathrm{I}_{\mathrm{mn}}(\mathrm{r}) \rightarrow \Psi_{\mathrm{mn}}\left(\mathrm{r}_{\mathrm{j}}\right) \quad \mathrm{j}: 1, \ldots, \mathrm{I} \cdot \mathrm{I}
$$

RSF has confirmed, with greater efficiency, the multiple helfcity results of RS3 (Fig. 3) and the single helicity results of the MASS code (Fig. 1).

As long as the solution is dominated by a moderate number of $(m ; n)$ modes, this representation is considerably more natural and economical than grids in $\theta$ and $\zeta$. This certainly holds in the early stages of a calculation, where very low mode numbers dominate. In the later stages of some multihelicity calculations, short wavelength components of the solution become important. Beyond a certain point, both finite-difference and finite-series representations become inadequate. 


\subsubsection{Equations}

As in Ref. 10, a two-step algorithm is proposed:

$$
\begin{aligned}
& u^{t+\Delta t / 2}=u^{t}+\frac{\Delta t}{2} s_{1} \\
& \tilde{\psi}^{t+\Delta t / 2}=\tilde{\psi}^{t}+\frac{\Delta t}{2} s_{2} \\
& u^{t+\Delta t}=u^{t}+\Delta t s_{3} \\
& \tilde{\psi}^{t+\Delta t}=\tilde{\psi}^{t}+\Delta t s_{4} .
\end{aligned}
$$

The four source terms $\left(S_{1}\right)$ are determined from Eqs. (1) and (2) with the following constraints:

(1) $S_{3}$ and $s_{4}$ are evaluated at $t+\Delta t / 2$ to give a second-order accurate timestep.

(2) All nonlinear terms are evaluated explicitly to avoid solving very large matriy problems.

(3) The U equation is treated explicitly. A stability analysis (similar to that in ket. 6 for the MASS code) shows that the choice of explicit $U$ equation and partly implicit $\Psi$ equation is more efficient than fully implicit or fully explicit algorithms.

An approximate von Neumann stability analysis is performed on the equations to determine the best form for the time advancement scheme within these constraints. The result is

$$
\begin{aligned}
s_{1} & =\nabla_{r} \Phi^{t} \nabla_{\theta} U^{t}-\nabla_{\theta} \Phi^{t} \nabla_{r} U^{t} \\
& -s^{2}\left[\frac { 1 } { q } \left(-r \nabla_{\theta} \tilde{J}_{\zeta}^{t}+q \frac{\partial \sim J_{\zeta}^{t}}{\partial \zeta}-\nabla_{\theta} \tilde{\Psi}^{t} \frac{d}{d r} J_{\zeta O}\right.\right. \\
& \left.+\nabla_{r} \tilde{r}^{t} \nabla_{\theta} \tilde{J}_{\zeta}^{t}-\nabla_{r} \tilde{J}_{\zeta}^{t} \nabla_{\theta} \tilde{\Psi}^{t}\right], \\
s_{2} & =\frac{r}{q} \nabla_{\theta} \Phi t-\frac{\partial}{\partial \zeta} \Phi^{t}+\tilde{n J}_{\zeta}^{t+\Delta t / 2}-\nabla_{r} \tilde{\Psi}^{t} \nabla_{\theta} \Phi^{t}+\nabla_{\theta} \tilde{\Psi}^{t} \nabla_{r} t,
\end{aligned}
$$




$$
\begin{aligned}
S_{3} & =\nabla_{r} \Phi^{t+\Delta t / 2} \nabla_{\theta} U^{t+\Delta t / 2}-\nabla_{\theta} \Phi^{t+\Delta t / 2} \nabla_{r} U^{t+\Delta t / 2} \\
& -s^{2}\left[\frac{1}{q}\left(-r \nabla_{\theta} \tilde{J}_{\zeta}^{t+\Delta t / 2}+q \frac{\partial}{\partial \zeta} \tilde{J}_{\zeta}^{t+\Delta t / 2}\right)-\nabla_{\theta} \tilde{\Psi}^{t+\Delta t / 2} \frac{d}{d r}{ }_{\zeta O}\right. \\
& \left.+\nabla_{r}{ }^{\tau}{ }^{t+\Delta t / 2} \nabla_{\theta} \tilde{J}_{\zeta}^{t+\Delta t / 2}-\nabla_{r} \tilde{J}_{\zeta}^{t+\Delta t / 2} \nabla_{\theta} \tilde{\Psi}^{t+\Delta t / 2}\right],
\end{aligned}
$$

and

$$
\begin{aligned}
s_{4} & =\frac{r}{q} \nabla_{\theta}{ }^{t+\Delta t / 2}-\frac{\partial}{\partial \zeta} \Phi^{t+\Delta t / 2}+\tilde{n J}_{\zeta}^{t+\Delta t / 2} \\
& -\nabla_{r} \tilde{\Psi}^{t+\Delta t / 2} \nabla_{\theta} \Phi^{t+\Delta t / 2}+\nabla_{\theta} \tilde{\Psi}^{t+\Delta t / 2} \nabla_{r}{ }^{t+\Delta t / 2},
\end{aligned}
$$

where

$$
\mathrm{U} \equiv \frac{1}{\mathrm{r}} \nabla_{\mathrm{r}}\left(\mathrm{r} \nabla_{\mathrm{r}} \Phi\right)+\nabla_{\theta}^{2} \Phi
$$

and

$$
J_{\zeta} \equiv \frac{1}{r} \nabla_{r}\left(r \nabla_{r} \Psi\right)+\nabla_{\theta}^{2} \Psi,
$$

and where

$$
\nabla_{\theta} \equiv \frac{1}{r} \frac{\partial}{\partial \theta}
$$

and

$$
\nabla_{\mathrm{r}} \equiv \frac{\partial}{\partial \mathrm{r}}
$$

Each of the field quantities in Eqs. $(22)-(28)$ (i.e., $J, \Phi, \tilde{J}_{\zeta}, \tilde{\Psi}$ ) is then expanded as in Eq. (10). Each equation is multiplied by $\cos (m \theta+n \zeta)$ or $\sin (m \theta+n \zeta)$ and integrated with respect to $\theta$ and $\zeta$. This has the effect of projecting out a single mode on the left-hand side of $\mathrm{Eq} \cdot(22)$. The mode numbers for linear source terms agree with tho quantity being advanced; for example, 


$$
\frac{\Psi_{m n}^{t+\Delta t / 2}-\Psi_{m n}^{t}}{\Delta t / 2}=\frac{r^{q}}{q} \nabla_{\theta} \Phi_{m n}^{t}+\cdots \cdot .
$$

If a total of $L$ modes are included in the calculation, then in each half of the step, there are 2L equations to be advanced. Each of the 12 nonlinear terms becomes a convolution of amplitudes; for example,

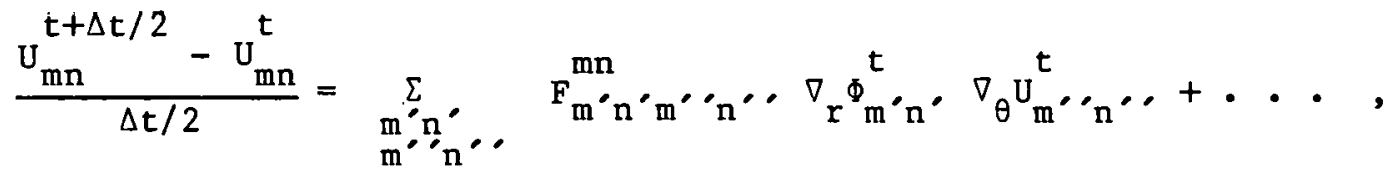

where $F$ is a sparse matrix whose elements have values \pm 1 or 0 .

For each timestep, three tridiagonal matrix problems must be solved:

(1) for $\Phi^{t}$ using Eq. (27),

(2) for $\Psi^{t+\Delta t / 2}$ (dine to the $\eta_{\zeta} t+\Delta t / 2$ t.e.rm) in $E q \cdot(2 /)$, and

(3) for $\Phi^{t+\Delta t / 2}$ using Eq: (27).

These are not a very time-consuming part of the timestep.

In RSF, Eqs. (22)-(28) are solved using the boundary conditions as stated earlier. It has not been necessary to employ any techniques to suppress numerical difficulties. Except in the most pathological cases, the conde has performed quite accurntely. In cylindicical geometry the origin $(r=0)$ can sometimes be difficult to treat numerically. Such difficulties are avoided here because all functions are scalar (rather than vector or tensor) fields and because regularity at the origin is particularly easy to impose in terms of individual modes. For $m \neq 0$ components, the scalar functions must go to zero at 
$r=0$. For $m=0$ components, the first derivative with respect to $r$ must vanish at $r=0$.

The entire calculation is carried out in terms of the modes ( $\Psi_{\mathrm{mn}}$, $\mathrm{U}_{\mathrm{mn}}$, etc.). Fourfer transforms back to $(r, \theta, \zeta)$-space are used only for generating certain types of output, never for solving the PDEs thems elves.

\subsubsection{Choice of Modes}

Cases in which only a single mode is perturbed are called single helicity cases. This is because the nonlinear terms generate only modes of the same helicity, $\mathrm{p}=\mathrm{m} / \mathrm{n}$, as the perturbed mode. Thus, Eq. (10) reduces to

$$
\Psi(r, \theta, \zeta, t)=\Psi_{0, o}(r)+\sum_{n=n_{m i n}}^{\infty} \Psi_{n p, n}(r) \cos [(p \theta+\zeta) n] .
$$

It is necessary to truncate the series at some finite value $n=N$. Single helicity calculations have two general types of solutions. In saturation cases, the solution'goes to a time-independent final state in such a way that the magnitudes of the amplitudes drop of $f$ rapidly with $n$ at all times during the calculation. ${ }^{9}$ The second class of single helicity cases, where magnetic reconnection occurs, results in a time-independent final state dominated by the $(m=0 ; n=0)$. However, there is a point in the evolution at which it is necessary to include a substantial number of modes. In either case, the number of modes required in $\mathrm{RSF}$ is considerably smaller than the number of grid points required in the MASS code (Figs. 1 and 4 ). 
For multihelicity cases, in which modes of unequal helicity are perturbed, the choice of modes is not so simple. One could argue that the only criterion should be to keep modes of long wavelength. This suggests a condition such as

$$
\mathrm{n} \leqslant \mathrm{N} \text { and } \mathrm{m} \leqslant \mathrm{M}
$$

or perhaps

$$
n^{2}+m^{2} \leqslant M^{2}+N^{2}
$$

Experience has shown that these are very inefficient choices because they include many unimportant modes. There is a tendency for the modes that are not resonant, for which $p=m / n$ does not equal $q(r)$ anywhere in the plasma, to be unimportant. During the early stages of evolution, the one or two modes that are linearly unstable completely dominate. Considering this, an ad hoc ordering scheme has been devised that can be used to determine an efficient choice of modes. Consider a case in which the $(m=2 ; n=1)$ and $(m=3 ; n=2)$ modes are linearly unstable and in which the $(m=2 ; n=1)$ mode is significantly larger than the $(m=3 ; n=2)$ mode throughout the calculation. Assume that

$$
\begin{aligned}
& \Psi_{00} \cdot 1, \\
& \Psi_{21} \sim \delta,
\end{aligned}
$$

and

$$
\Psi_{32} \sim \delta^{2},
$$

where $\delta$ is a small parameter. 
When two modes interact, they directly generate modes with mode numbers that are the sum and difference: $\left(m_{1} ; n_{1}\right)$ and $\left(m_{2} ; n_{2}\right)$ directly generate $\left(m_{1}+m_{2} ; n_{1}+n_{2}\right)$ and $\left(m_{1}-m_{2} ; n_{1}-n_{2}\right)$. This leads to the following ordering:

$$
\begin{aligned}
& O\left(\delta^{0}\right): \quad \Psi_{0,0} \\
& 0\left(\delta^{1}\right): \quad \Psi_{2,1} \\
& 0\left(\delta^{2}\right): \Psi_{3,2} \quad \Psi_{4,2} \\
& 0\left(\delta^{3}\right): \quad \Psi_{1,1} \Psi_{5,3} \quad \Psi_{6,3} \\
& 0\left(\delta^{4}\right): \quad \Psi_{1,0} \Psi_{6,4} \quad \Psi_{7,4} \quad \Psi_{8,4} \\
& 0\left(\delta^{5}\right): \quad \Psi_{3,1} \quad \Psi_{4,3} \quad \Psi_{8,5} \quad \Psi_{9,5} \quad \Psi_{10,5} \\
& 0\left(\delta^{6}\right): \quad \Psi_{2,2} \quad \Psi_{5,2} \quad \Psi_{9,6} \Psi_{10,6}{ }_{11,6}{ }_{12,6}^{\Psi_{1}} \\
& 0\left(\delta^{7}\right): \quad \Psi_{0,1} \quad \Psi_{7,3} \quad \Psi_{7,5} \quad \Psi_{11,7} \Psi_{12,7} \quad \Psi_{13,7} \quad \Psi_{14,7}
\end{aligned}
$$

It is natural to truncate the series at some order; thus choices of 7 , $11,16,22$, or 29 modes are reasonable. Runs have been made with up to 79 modes.

Although ordering pyramids, like that above, have been remarkably ouccessful in predicting the modes that are important for numerical calculations, they in no way constitute a proof that other modes are not important. This mist be tested by making runs using different modes.

To produce Fig. 5, the modes $(0 \leqslant n \leqslant 5 ; 0 \leqslant m \leqslant 5)$ were used. The magnetic energy (ME) of each mode was determined late in the run. Modes for which $-\log$ ME is large are relatively unimportant. Each of the 36 modes is assigned an order according to three different schemes: (1) using the ordering pyramid above;

(2) following the above procedure, but assuming $\Psi_{3,2}=0\left(\delta^{1}\right)$ 
and modifying the pyramid accordingly; or

(3) setting the order to $\min (m, n)$.

The whole purpose of the ordering scheme is to have a reasonably good predictor of mode importance. Figure 5 shows that for this case scheme 1 is the best predictor of mode size. Scheme 2 is slightly worse, and scheme 3 shows only slight correlation between order and mode size. When the ordering.pyramid is employed, the convergence of the solution with the number of modeo is rapid (Fig. 6 ).

With RSF it is possible to exclude individual modes selectively in order to assess their importance. This facility has proved useful in understanding major disruption cases. ${ }^{15}$ In a pure finite-difference scheme, such as RS3, this technique cannot be used. RSF also has the advantage of making it possible to study the linear behavior of high-numbered modes inexpensively and accurately •

When the $n$ values of the modes in a calculation are multiplied by a positive luleges $I$ dind when $q+q / I$, then the solutions are unchanged. It is necessary for the solutions to possess this property, which provides a check against certain programming errors.

\subsubsection{Convolution}

Each timestep requires 12 convolutions [Eq. (29)]. This is the most rime-consuming part of the calculation. If the total number of mọtes is $L$ and the number of radial grid points is $J$, then for each of the 12 convolutions there are $\mathrm{L} \times \mathrm{J}$ possible left-hand sides. For each of these, the summation will have on the order of L nonzero terms. Since each calculation requires a large number $\left(\sim 10^{3}\right.$ to $\left.\sim 10^{5}\right)$ of timesteps, it is desirable to set up as much of the convolution as 
possible at the start of the run. Assume, in Eq. (29), that the derivatives have been done, giving

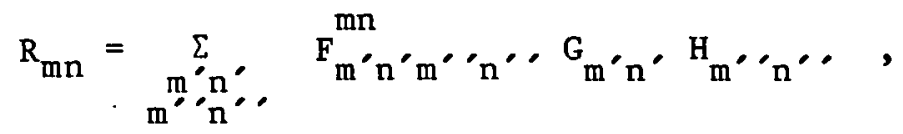

where the summation includes only the terms chosen by the ordering scheme. Further, assume that the set of modes included in the run forms an ordered list:

$$
\left(\mathrm{m}_{\ell} ; \mathrm{n}_{\ell}\right) \quad \ell=1,2, \ldots, \mathrm{L} \quad .
$$

Each mode can be identified by an $\ell$ subscript rather than the double mn notation:

$$
\mathrm{R}_{\ell}=\ell_{\ell, \ell^{\prime}}^{\Sigma}, \mathrm{F}_{\ell}^{\ell} \ell^{\prime}, \mathrm{G}_{\ell}, \mathrm{H}_{\ell} \cdots
$$

At the start of a run, two subscript arrays are prepared, allowing the convolution to take the following form (in FORTRAN):

$$
\begin{array}{rl} 
& \mathrm{R}=0 . \\
\mathrm{DO} 100 \mathrm{LP}=\mathrm{LPMIN}, \text { LPMAX } \\
100 & \mathrm{R}=\mathrm{R}+\mathrm{G}(\mathrm{LG}(\mathrm{LP})) * \mathrm{H}(\mathrm{LH}(\mathrm{LP}))
\end{array}
$$

LPMIN and LPMAX are set to pick out the parts of LG and LH that are needed to calculate the current element of $R$. This technique avolds all of the zero contributions to the convolution. Unfortunately, this style of DO loop is very inefficient on the CRAY-1 computer. Since nearly all of our production runs have been performed on a CRAY-1, it is neressary to look for another way to do the convolution. Since 
there is a fintte-difference grid in minor radius, each of the field quantities is stored as, for example,

$\operatorname{PSI}(j, \ell)$,

where $j$ labels the grid point and $\ell$ labels the mode. Since the convolution operation must be done at each grid point, the $j$ loop can be brought inside the $\&$ loop, giving

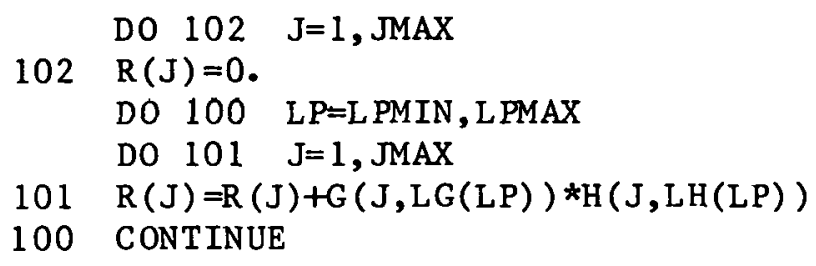

This generates a very efficient vector code on the CRAY-1.

\section{3 .4 Timestep}

When Eqs. (23)-(26) are linearized, an approximate von Neumann stability analysis gives

$$
\Delta \varepsilon_{0} \leqslant \frac{2}{s \operatorname{Max}|n-m / q|}
$$

where the Max is over all $(m ; n)$ pairs in the calculation and over all values of $q$ in the plasma. Runs with high values of $m$ and $n$ require a smaller timestep. This is analogous to the dependence on grid spacing in the MASS code and in RS3. Note that the spacing in minor radius does not appear in Eq. (34). This is fortunate, since in many cases 100 to 300 grid points are used. 
The initial timestep size is given by

$\Delta t \equiv \Delta t_{0} \cdot$ DTO ,

where DTO is an input variable that is set to a value somewhat less than 1 (usually $0.2 \leqslant$ DTO $\leqslant 0.8$ ) so that the initial timestep is somewhat smaller than that required by the stability analysis. As nonlinear effects become important, it proves necessary to reduce the timestep. A procedure for decreasing it during a run has been developed. It has a rather weak theoretical basis, but in practice it works quite well. When a numerical instability starts to grow, it can usually be seen as a short wavelength oscillation in the modes. To detect the oscillation early in its development, it is advisable to look at a sensitive quantity. $J_{\zeta}$ is more sensitive to the numerics than is $\Psi$ since $J_{\zeta}$ is the second derivative of $\Psi$. Since $J_{\zeta 00}$ is always present, we choose that component. In particular, whenever the number of oscillations (as a function of $r$ ) of $\left(J_{\zeta}\right)_{00}$ increases, the timestep is reduced by a factor DTRED, which is usually set to 0.8 . There are certainly some cases in which this fails, usually because the timestep is unnecessarily lowered, but in most cases this procedure works well.

A code, RSFMOL, has been developed in which this timestepping procedure is replaced by a method-of-lines technique. 21 Severa1 standard ordinary differential equation (ODE) solvers that determine their own timesteps have been used. 22 This appears to be an expensive technique; it does not pay for itself until very late in a run, where, using our standard technique, DTRED has unnecessar1ly reduced the timestep. 
Frequently it is difficult to estimate. in advance how long a computer run will be needed in a given case. In many cases, runs have not progressed far enough, so a facility to continue runs has been built into RSF and the other initial value codes. An unformatted file of data is saved after each run. It contains sufficlent information to continue the run.

\subsubsection{Grid}

Tearing modes are localized in minor radius. This localization becomes more severe at high values of $\mathrm{s}$ or $\mathrm{m}$. Our initial value codes allow a nonunfform grid to be spectfied. In single helicity cases, this can be put to significant advantage by putting a high concentration of points near the resonant surface. For multihelicity cases with many resonant surfaces, the advantage is considerably reduced. Often such runs are made with uniform grids. The finite-difference formulas for first and second derivatives are three-point formulas that take unequal spacing into account.

\subsubsection{Sources of Numerical Error}

There are five sources of numerical error:

(1) truncation of the Infinite sum of modes;

(2) truncation error due to the finite grid in $r$;

(3) truncation error due to finite timestep;

(4) roundoff error; and

(5) numerical instability. 
For multihelicity runs, the results converge as the number of modes $\mathrm{L}$ is increased (Fig. 6). The time at which numerical error becomes significant comes later in the run for larger $L$. This reflects the fact that, as time progresses, more and more modes become important.

At small values of $S\left(e \cdot g \cdot, S \simeq 10^{4}\right), 100$ to 200 grid points are adequate, while at larger values (e.g., $S \simeq 10^{6}$ ), 200 to 300 points are usually needed. This corresponds to $\Delta r \simeq 10^{-3}$ in the vicinity of important singular surfaces. A case must be run with at least two different grid sizes before it is considered verified. The inset in Fig. 7 shows the toroidal current $J_{\zeta}$ late in a run at $S=10^{5}$. An enlargement of the region with small wavelength oscillations shows that the spikes are real; they are well-reproduced on two different grids. The magnetic island width of the $(m=3 ; n=2)$ mode is rather sensitive to numerical error. It is plotted in Fig. 8 as a function of time for several grid sizes, again at $S=10^{5}$. Convergence to the solution is quite evident.

Within the numerical stability constraint of Eq. (34), the timestep has been varied to study the effects of the finite-difference expression for the time derivative. No detectable effects have been seen. Evidently, numerical stability is the more restrictive constraint.

RSF is normally run in single precision on the CRAY-1. Floating-point format uses 48 bits $\left(14^{+}\right.$decimal digits $)$for the mantissa. The floating point precision has been varied, showing that roundoff error is not significant. 
The control of numerical instability is accomplished in the ad hoc fashion described in sect. 3.3.4. As the inftial timestep is increased, there is a sharp onset of short wavelength numerical oscillations. It is presumed that the absence of such oscillations indicates the lack of significant numerical instability.

\subsubsection{Output}

At specified times during a run, printed or plotted information reflecting the current state of the calculation is output. What follows is a selective summary of that information.

Plots are provided of $\Psi, J_{\zeta}, \tilde{\Psi}, \tilde{J}_{\zeta}$, and the toroidal electric field across a horizontal diameter of the plasma. For each mode, $\Psi_{m n}$, $\mathrm{J}_{\zeta \mathrm{mn}}$, and $\Phi_{\operatorname{mn}}$ are plotted as functions of $r$. For each resonant helicity, the function

$$
\Psi_{\mathrm{p}}^{*}=-\mathrm{p}\left[\Psi_{00}+\sum_{\mathrm{p}=\mathrm{n} / \mathrm{n}} \Psi_{\mathrm{mn}} \cos (\mathrm{m} \theta+\mathrm{n} \zeta)\right]-\frac{1}{2}\left(\mathrm{r}^{2}-1\right)
$$

is generated. This function in the single helicity approximation is the helical flux function. Contours of constant $\Psi_{p}^{\text {th }}$ are plotted for the $\zeta=0$ plane. These plots show the magnetic islands for each helicity as if the other helicities were absent. An arrow plot of the poloidal velocity in the $\zeta=0$ plane is also generated. At the end of each run, plots appear of the island widths, limiter voltage, and plasma self-inductance, all as functions of time.

The printed output starts by reflecting the input data and listing the values of all initfalization and control variables whether they are default values or not. For each grid point, the radius $r$ is given, 
along with the local grid spacing $\Delta r$ and the equilibrium quantities $q(r), J_{\zeta}(r), n(r)$, and $\Psi(r)$.

Periodically during a run, the step number, time, timestep size, limiter voltage, and total plasma current are given. For the constant current boundary condition, the limiter voltage is given by

$$
V_{L}=\frac{-\pi^{2}}{I}\left[\frac{d}{d t}\left(\varepsilon_{M}+\varepsilon_{K}\right)+Q\right],
$$

where I is the total plasma current,

$$
I=\int r d r d \theta J_{\zeta}
$$

and $\varepsilon_{M}$ is the total magnetic energy,

$$
\varepsilon_{M}=\sum_{m, n}\left(\varepsilon_{M}\right)_{m n}=2 \pi^{2} \sum_{m, n} \int r d r\left[\left(\nabla_{r} \Psi_{m n}\right)^{2}+\left(\nabla_{\theta} \Psi_{m n}\right)^{2}\right]
$$

For each mode, the kinetic energy

$$
\left(\varepsilon_{\mathrm{K}}\right)_{\mathrm{mn}}=\frac{2 \pi^{2}}{\mathrm{~s}^{2}} \int \mathrm{r} \mathrm{dr}\left[\left(\nabla_{\mathrm{r}} \Phi_{\mathrm{mn}}\right)^{2}+\left(\nabla_{\theta} \Phi_{\mathrm{mn}}\right)^{2}\right],
$$

the magnetic energy $\left(\varepsilon_{\mathrm{M}}\right)_{\mathrm{mn}}$, the maximum of $\Psi_{\mathrm{mn}}(r)$, and the average of $\Psi_{\mathrm{mn}}(r)$ are given along with the instantaneous exponential growth rate of each. We also print $\varepsilon_{\mathrm{M}}, \dot{\varepsilon}_{\mathrm{M}}, \varepsilon_{\mathrm{K}}, \dot{\varepsilon}_{\mathrm{K}}, \mathrm{Q}, \mathrm{F}$, and a measure of energy conservation [see Eq. (18)]: 


$$
\text { DIFF } \equiv \frac{\dot{\varepsilon}_{K}+\dot{\varepsilon}_{M}+Q+F}{\dot{\varepsilon}_{K}+\dot{\varepsilon}_{M}} \text {, }
$$

where "dot" implies time derivative.

Two other global quantities, which are conserved in ideal MHD, are evaluated. The first is

$$
K_{0} \equiv \int \vec{A} \cdot \vec{B} d V \text {, }
$$

where $\vec{A}$ is the vector potential of the magnetic field. This quantity has been useful, for example, in analyzing hollow current profile cases. $^{14}$ In terms of our dimensionless quantities, we have

$$
\overline{\mathrm{K}}_{0}=4 \pi^{2} \int_{0}^{1} r \mathrm{dr}\left(\frac{1}{2} r \frac{\partial}{\partial \mathbf{r}} \psi_{00}-\psi_{00}\right) .
$$

The second is the invariant

$$
\mathrm{K}_{1} \equiv \int \overrightarrow{\mathrm{v}} \cdot \overrightarrow{\mathrm{B}} \mathrm{dV}
$$

Note that with the usual initial conditions, this evaluates to zero oince $\Psi \sim \cos (m \theta+n \zeta)$ and $I \sim \sin (m \theta+n \zeta)$. Using the full MHD equations $^{10}$ [from which Eqs. (1) and (2) are derived], one finds that the time derivative of $\mathrm{K}_{1}$ is equal to the sum of two terms. The first is a surface term that goes to zero when $B_{\perp}=v_{1}=0$ at the wall. The second is proportional to the resistivity. Starting from the reduced Eqs. (1) and (2), one gets a dimensionless "invariant" 


$$
\begin{aligned}
\overline{\mathrm{K}}_{1} & \equiv \frac{\mathrm{K}_{1}}{\left(-\varepsilon \mathrm{B}_{\zeta \mathrm{O}}\right)} \\
& =\int \mathrm{d} V \vec{\nabla}_{\perp} \phi \cdot \vec{\nabla}_{\perp}^{\Psi}=\int \mathrm{d} \vec{s} \cdot \vec{\nabla}_{\phi \Psi}-\int \mathrm{d} V \mathrm{U} \Psi
\end{aligned}
$$

When $\Phi=0$ at the wall, this becomes

$$
\begin{aligned}
& \partial_{t}\left(\overline{\mathrm{K}}_{1}+\int \overrightarrow{\mathrm{d} s} \cdot \vec{\nabla} \Phi \Psi\right)=\mathrm{s}^{2} \int \mathrm{d} s\left(\frac{1}{2} \mathrm{~J}_{\zeta} \frac{\partial}{\partial \theta} \Psi^{2}+\frac{\partial}{\partial \zeta} \Psi \frac{\partial}{\partial r} \Psi\right) \\
& \quad+\int \mathrm{dVU}\left(n \mathrm{~J}_{\zeta}-\mathrm{E}_{\zeta}^{\mathrm{W}}\right)
\end{aligned}
$$

If also $\Psi=0$ at the wall, then

$$
\partial_{t} \bar{K}_{1}=\int d V U\left(E_{\zeta}^{W}-n J_{\zeta}\right)=\int d V U \tilde{U J}_{\zeta}
$$

For each helicity where an island width $\mathrm{w}_{\mathrm{p}}$ can be measured, the radial position of the island center, of the inside and outside edges of the island, and of $\dot{W}_{\mathrm{p}}$ are all given. For this purpose, $\Psi_{\mathrm{p}}^{*}$ is calculated as in Eq. (35). 
38

Blank 


\section{COMPARISON OF CODES}

The reduced MHD equations [Eqs. (1) and (2)] are solved by the authors with a system of codes (Table 1). The first six codes are for circular cylinder geometry. LINEAR and DELSOL solve the linear (1-D) problem. Using a $\Delta^{\prime}$ technique, DELSOL can provide a rather reliable estimate of saturated island width in addition to linear growth rate. The MASS code was developed to study the nonlinear evolution of single helicity (2-D) perturbations. In RS3, the full 3-D, nonlinear, initial-value problem is solved. Now, however, both of these codes are superseded by RSF, which is superior in efficiency for both 2-D and 3-D problems. Today, the MASS code and RS3 are primarily used to provide an independent verification of RSF results.

Both RSF and RS3 are well optimized for the CRAY-1 computer. However, it is difficult to compare the run times properly. As a function of the number of modes $L$, RSF converges rapidly to an accurate solution. RS3 requires a very large grid (larger than $60 \times 38 \times 19$ ) to obtain the accuracy of an 11-mode RSF run for a typical 3-D, nonlinear run. From this case we estimate that RSF is at least two orders of magnitude faster than RS3 on average.

Using the method of lines, RSFMOL ${ }^{21}$ also verifies the RSF results. Codes have also been developed for toroidal geometry. ${ }^{16,17}$ LOBETO is similar to RSF, but it uses a toroidal geometry flux coordinate system. The linear LOBETO results indicate that, although all modes with equal n couple, a good approximation to the linear eigenfunction and eigenvalue can be obtained by including only two modes. This leads to the two-mode $\Delta^{\prime}$ calculator, TORDEL. 
The magnetic field configurations in the nonlinear codes can be further analyzed at any point in time with the code FL. Using the integrator $D E,^{23}$ it is capable of following a magnetic field line many times around the torus. Plots of where a single field line crosses a given poloidal plane $(\zeta=$ constant) are very useful for understanding the complexity of the field. When regions of stochastic magnetic field are present, FL is used to calculate the maximal Lyapunov number, the diffusion coefficients for the stochastic region, and the poloidal area of the region. 


\section{REFERENCES}

1. E. P. Gorbunov and K. A. Razumova, At. Energy 15, 363 (1963) [Sov. At. Energy 15, 1105 (1963)]; L. A. Artsimovich, S. V. Mirnov, and V. S. Strelkov, At. Energy 17, 170 (1964) [Sov. At. Energy 17, $886 \quad(1964)]$; L. A. Artsimovich, V. A. Venshkov, A. V. Glukhov, E. P. Gorbunov, V. S. Zaveryzev, S. E. Lysenko, S. V. Mirnov, I. B. Semenov, and V. S. Strelkov, in Plasma Physics and Controlled Nuclear Fusion Research, Vol. I, p. 443 (1971); S. von Goeler, W. Stodiek, and N. Sauthoff, Phys. Rev. Lett. 33, 1201 (1974); V. S. Vlasenkov et al., Nucl. Fusion Supp1. 1,1 (1975); L. A. Berry et a1., in Plasma Physics and Controlled Nuclear Fusion Research, Vol. I, p. 49 (1977); I. Hutchinson, Phys. Rev. Lett. 37, 388 (1976); D. B. Albert and A. H. Morton, Nucl. Fusion 17, 863 (1977); S. V. Mirnov and I. B. Semenov, in Plasma Physics and Controlled Nuclear Fusion Research, Vol. I, p. 291 (1977); N. R. Sauthoff, S. von Goeler, and W. Stodiek, Nucl. Fusion 18, 1445 (1978); K. Toi, S. Itoh, K. Kadota, K. Kawahata, N. Noda, K. Sakurai, K. Sato, S. Tanahashi, and S. Yasue, Nucl. Fusion 19 , 1643 (1979).

2. B. V. Wadde11, M. N. Rosenbluth, D. A. Monticello, and R. B. White, Nuc1. Fusion 16, 528 (1976).

3. D. Biskamp and $\mathrm{H}$. Welter, in Plasma Physics and Controlled Nuclear Fusion Research, Vol. I, p. 579 (1977).

4. R. B. White, D. A. Monticello, M. N. Rosenbluth, and B. V. Waddell, in Plasma Physics and Controlled Nuclear Fusion Research, Vol. I, p. $569(1977)$.

5. R. B. Wh1te, D. A. Monticello, M. N. Rosenbluth, and B. V. Wadde11, Phys. Fluids 20, 800 (1977).

6. B. V. Wadde11, M. N. Rusenblutl, D. A. Monticello, R. B. White, and B. Carreras, in Theoretical and Computational Plasma Physics, p. 79 (1978).

7. G. L. Jahns, Mario Soler, Bruce V. Wadde11, James D. Callen, and H. Richard Hicks, Nucl. Fusion 18, 609 (1978); B. V. Waddell, G. L. Jahns, J. D. Callen, and H. R. Hicks, Nucl. Fusion 18, 735 (1978).

8. B. V. Wadde $\perp$, B. Carreras, H. R. H1cks, J. A. Hulules, and D. K. Lee, Phys. Rev. Lett. 41, 1386 (1978).

9. B. Carreras, B. V. Waddell, H. R. Hicks, and S. J. Lynch, Phys. Rev. A 18, 735 (1978).

10. B. V. Waddell, B. Carrcrae, H. R. Hicks, and J. A. Holmes, Phys. Fluids 22, 896 (1979).

11. R. Carreras, B. V. Waddell, and H. R. Hicks, Nucl. Fusion 19, 1423 (1979). 
12. J. D. Callen, B. V. Wadde11, B. Carreras, M. Azumi, P. J. Catto, H. R. Hicks, J. A. Holmes, D. K. Lee, S. J. Lynch, J. Smith, M. Soler, K. T. Tsang, and J. C. Whitson, in Plasma Physics and Controlled Nuclear Fusion Research, Vol. I, p. 415 (1979).

13. J. A. Holmes, B. Carreras, H. R. Hicks, S. J. Lynch, and B. V. Wadde11, Nuc1. Fusion 19, 1333 (1979).

14. B. Carreras, H. R. Hicks, and B. V. Wadde11, Nucl. Fusion 19, 583 (1979).

15. B. Carreras, H. R. Hicks, J. A. Holmes, and B. V. Waddell, Phyc. Fluido 23, 1811 (1980).

16. B. Carreras, H. R. Hicks, and D. K. Lee, ORNL/TM-7281 (1980).

17. H. R. Hicks, B. A. Carreras, J. A. Holmes, D. Tetreault, G. Berge, J. P. Freidberg, and P. A. Politzer, to be published in Plasma Physics and Controlled Nuclear Fusion Research (1981).

18. H. P. Furth, J. Killeen, and M. N. Rosenbluth, Phys. Fluids 6 , 459 (1963) .

19. P. H. Rutherford, Phys. Fluids 16, 1903 (1973).

20. H. R. Strauss, Phys. Fluids $\underline{19}, 134$ (1976).

2.1. P. W. Caffncy, H. R. Hicks, and B. Carreras, ORNL/C SD/TM-133 $(1280)$.

22. P. W. Gaffney, paper presented at the Conference on Elliptic Partial Differential Equations, Santa Fe, New Mexico, June 30, 1980 (proceedings to be published); P. W. Gaffney, ORNL/CSD/TM-134 (1980).

23. L. F. Shampine and M. K. Gordon, Computer Solution of Ordinary Differential Equations: The Initial Value Problem, W. H. Freeman and Co., San Francisco, 1975. 
Table l. Tearing mode computer codes currently in use at ORIR,

\begin{tabular}{|c|c|c|c|c|c|}
\hline Name & Characteristics & $\begin{array}{l}\text { Geometry/ } \\
\text { dimensions }\end{array}$ & $\begin{array}{l}\text { Numerical } \\
\text { techn1que }\end{array}$ & Calculates & Comments \\
\hline L IUEAR & $\begin{array}{l}\text { Inear Intifial- } \\
\text { Jalue code }\end{array}$ & $\begin{array}{l}\text { Cylindrical/l } \\
\text { (single } \\
\text { hellcity) } \\
\end{array}$ & $\begin{array}{l}\text { Finite difference } \\
\ln r\end{array}$ & $\Psi_{\mathrm{mn}}(r, t)$ & $\begin{array}{l}\text { Used In profile } \\
\text { explorations }\end{array}$ \\
\hline DELSOL & $\begin{array}{l}\text { Linear boundary } \\
\text { zondition code }\end{array}$ & $\begin{array}{l}\text { Cylindrical/1 } \\
(\text { single } \\
\text { helicity) }\end{array}$ & $\begin{array}{l}\text { Finite difference } \\
\text { in } r\end{array}$ & $\Delta_{m n}^{\prime}$ and $W_{m n}$ & $\begin{array}{l}\text { Incorporated into Oak } \\
\text { Ridge transport code }\end{array}$ \\
\hline ILASS & $\begin{array}{l}\text { Nonlinear initial- } \\
\text { value code }\end{array}$ & $\begin{array}{l}\text { Cylindrical } / 2 \\
\text { (single } \\
\text { helicfty) }\end{array}$ & $\begin{array}{l}\text { Finite difference } \\
\text { in } r, \tau \equiv \zeta+(\mathrm{m} / \mathrm{n}) \theta\end{array}$ & $\begin{array}{l}\Psi(r, \tau, t) \\
\Phi(r, \tau, t) \\
T(r, \tau, t)\end{array}$ & $\begin{array}{l}\text { Ef ficiency low compared } \\
\text { with RSF, which effectively }\end{array}$ \\
\hline RS3 & $\begin{array}{l}\text { Nonlinear intital- } \\
\text { value code }\end{array}$ & $\begin{array}{l}\text { Cylindrical } / 3 \\
\text { (multiple } \\
\text { helictites) }\end{array}$ & $\begin{array}{l}\text { Finite difference } \\
\text { in } r, \theta, 5\end{array}$ & $\begin{array}{l}\Psi(r, \theta, 5, t) \\
\Phi(r, \theta, 5, t)\end{array}$ & $\begin{array}{l}\text { supersedes both these } \\
\text { codes }\end{array}$ \\
\hline RSE & $\begin{array}{l}\text { lonlinear inielal- } \\
\text { value ccde }\end{array}$ & $\begin{array}{l}\text { Cylindrical/3 } \\
\text { (multiple } \\
\text { helictites) }\end{array}$ & $\begin{array}{l}\text { Finite difference } \\
\text { ln } r \text {, Fourier } \\
\text { series in } \theta, \zeta\end{array}$ & $\begin{array}{l}\Psi(r, \theta, \zeta, t) \\
\$(r, \theta, \zeta, t) \\
T(r, \theta, \zeta, t)\end{array}$ & $\begin{array}{l}\text { Feedback stabllization } \\
\text { incorporated }\end{array}$ \\
\hline RSFMLL & $\begin{array}{l}\text { Nonlinear initial- } \\
\text { value code using } \\
\text { method of lines }\end{array}$ & $\begin{array}{l}\text { Cylindrical/3 } \\
\text { (multiple } \\
\text { helicities) }\end{array}$ & $\begin{array}{l}\text { Finite difference } \\
\text { in } r \text {, Fourier } \\
\text { series in } \theta, \zeta\end{array}$ & $\begin{array}{l}\Psi(r, \theta, \zeta, t) \\
\Phi(r, \theta, \zeta, t)\end{array}$ & $\begin{array}{l}\text { Less efficlent than } \\
\text { RSF except in very } \\
\text { nonlinear regime; } \\
\text { confirms RSF results. }\end{array}$ \\
\hline TORDEL & $\begin{array}{l}\text { Linear boundary } \\
\text { condition code }\end{array}$ & $\begin{array}{l}\text { Toroidal/l } \\
\text { (?-mode approx.) }\end{array}$ & $\begin{array}{l}\text { Finite difference } \\
\text { In } r\end{array}$ & $\begin{array}{l}W_{m, n}, W_{m+1, n} \\
\Delta_{m, n}^{\prime}, \Delta_{m+1, n}^{0}\end{array}$ & $\begin{array}{l}\text { Confirms linear } \\
\text { results of LOBETo }\end{array}$ \\
\hline LOBETO & $\begin{array}{l}\text { Honlinear initial- } \\
\text { value code }\end{array}$ & $\begin{array}{l}\text { Toroldal/3 } \\
\text { (multiple } \\
\text { heltcities) }\end{array}$ & $\begin{array}{l}\text { Finite difference } \\
\text { in } r \text {, Fourier } \\
\text { series in } \theta, \zeta\end{array}$ & $\begin{array}{l}\Psi(r, \theta, 5, t) \\
\Phi(r, \theta, 5, t)\end{array}$ & \\
\hline
\end{tabular}


44

klank 


\section{FIGURE CAPTIONS}

Fig. 1. The island width $W$ is shown as a function of time (a) for the MASS code with 8 and 24 points in the $\theta_{\mathrm{p}}$ grid. This is a typical $\mathrm{p}=2$ saturating island. When the grid is too coarse $(\mathrm{N}=8)$ there is a severe overshoot in island width. As $N$ is increased, the MASS code results approach the RSF results. In the coarse grid case $(\mathrm{N}=8)$ the time derivative of the width (b) considerably exceeds the estimate that comes from a $\Delta^{\prime}$ calculation.

Fig. 2. The maximum absolute value of the amplitude of $\Psi_{\mathrm{mn}}$ is plotted as a function of $n$ for the single helicity, $p=2$ MASs code runs shown in Fig. 1. The values shown were taken at a time corresponding to the peak in the island width. For the coarse grid run $(\mathrm{N}=8)$ the higher $\mathrm{n}$ modes are poorly resolved, resulting in anomalously large values of $\left|\Psi_{\mathrm{mn}}\right| \cdot$

Fig. 3. In the nonlinear regime, the instantaneous growth rate of the $(m=3 ; n=2)$ mode exhibits a strong peak just after the $p=3 / 2$ island overlaps with the $\mathrm{p}=2$ island. There is rough quantitative agreement between RS3 and RSF through this phase of the calculation.

Fig. 4. Single helicity $(\mathrm{m} / \mathrm{n}=2)$ case. The time dependence of the island width converges rapidly with the number of modes in RSF. The solid, dot-dashed, and long dashed curves correspond, respectively, to the mode selections $0 / 0,2 / 1,1 / 2 \quad(L=3) ; 0 / 0,2 / 1,1 / 2,6 / 3$ $(\mathrm{L}=4)$; and $0 / 0,2 / 1,4 / 2,6 / 3,8 / 4(\mathrm{~L}=5)$. The short dashed line is the MASS code result with MI $=24$. 
Fig. 5. For a 36-mode RSF case the magnetic energy is plotted as a function of "order." Order has been assigned to the 36 modes according to three different ad hoc schemes. In (a) there is the strongest correlation between order and magnetic energy. In (c) the order is a very poor predictor of the importance of a mode.

Fig. 6. 'The solution obtained with RSF converges rapidly as the number of modes is increased. Modes here were based on the ordering pyramid in the text. In this type of multihelicity run, smaller wavelengths become important as time goes on. Thus, the addition of more modes merely postpones the onset of numerical error.

Fig. 7. The toroidal current density viewed across a plasma diameter (inset) exhibits fine scale oscillations. The radial grid chosen must be sufficlently fine to accurately resolve them. A ciose inspection shows that for grid densities of the order of 200 , radial points are sufficient. The oscillations are seen to be insensitive to the locations of grid points.

Fig. 8. The $p=3 / 2$ magnetic island width becomes independent of radial grid density for $J \geq 100$. 

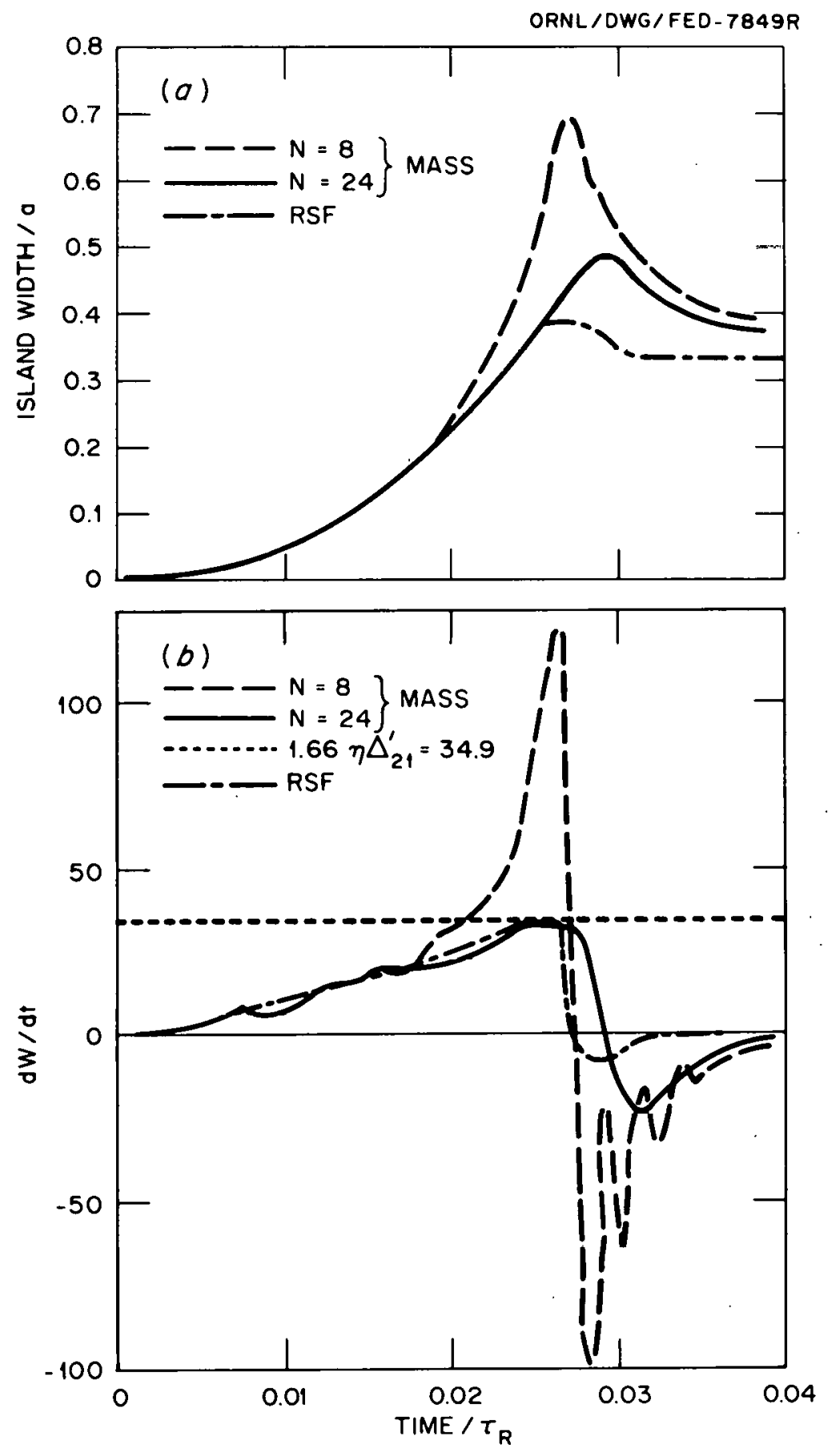

Fig. 1. 


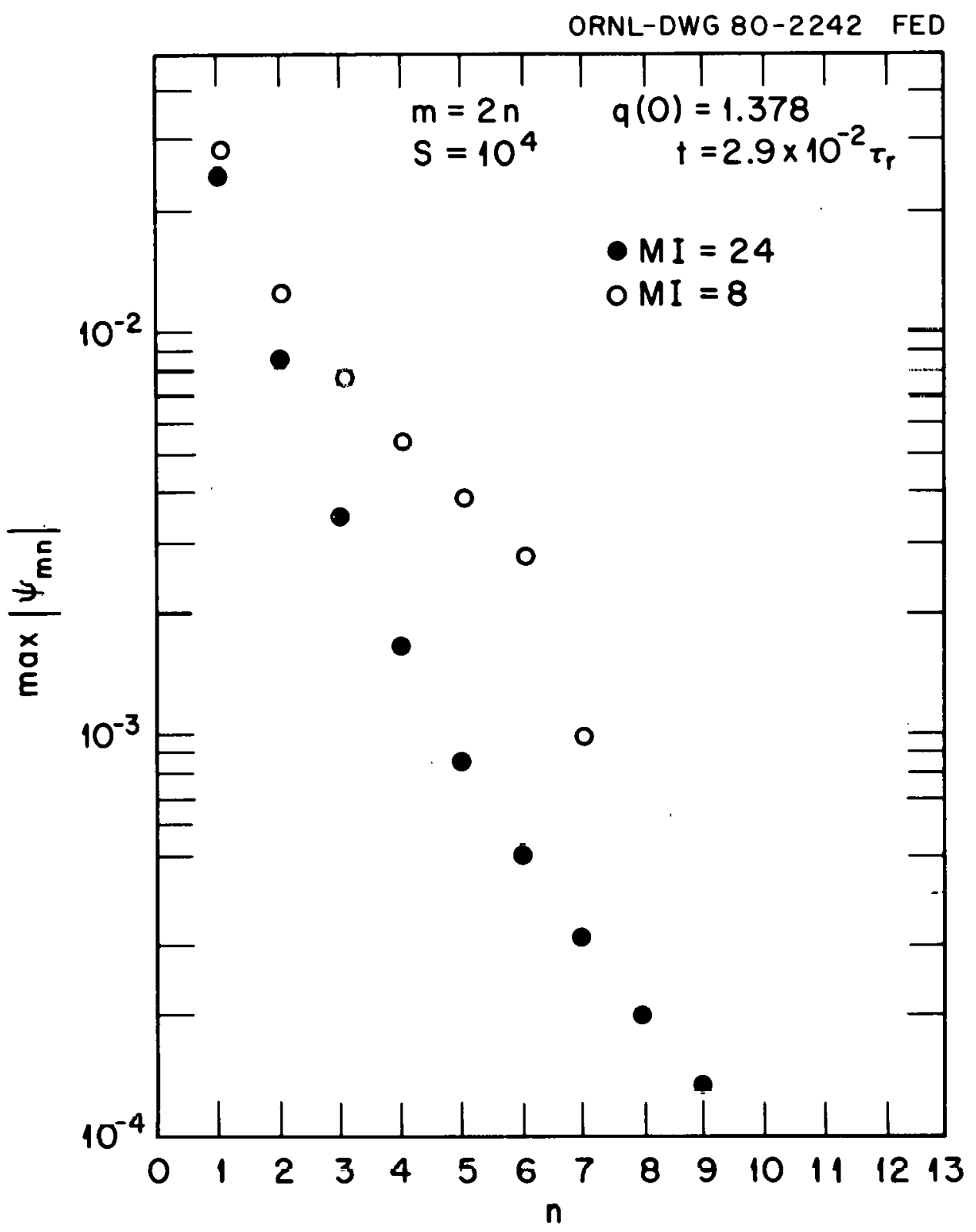

rig. 2. 


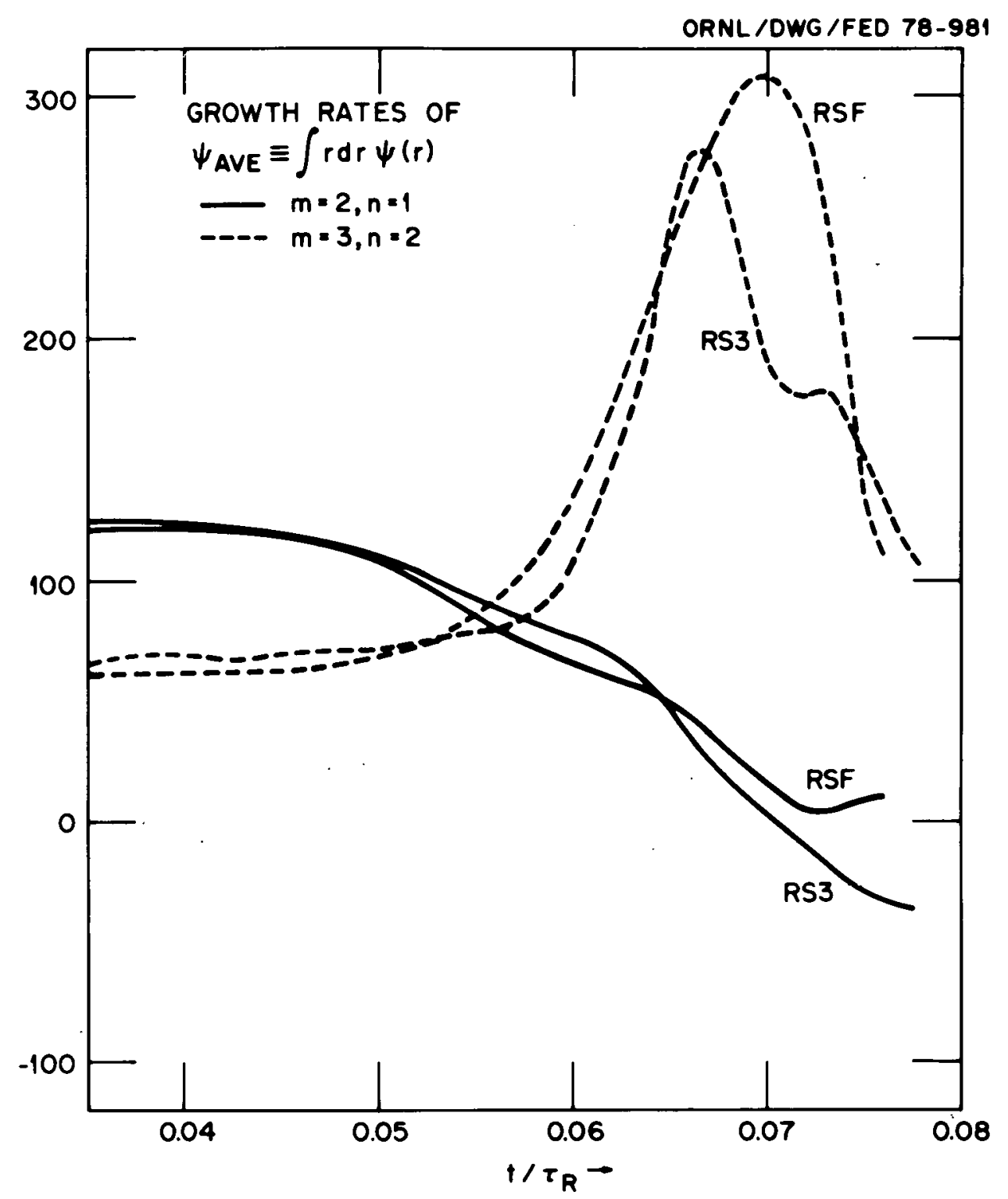

Fig. 3 . 


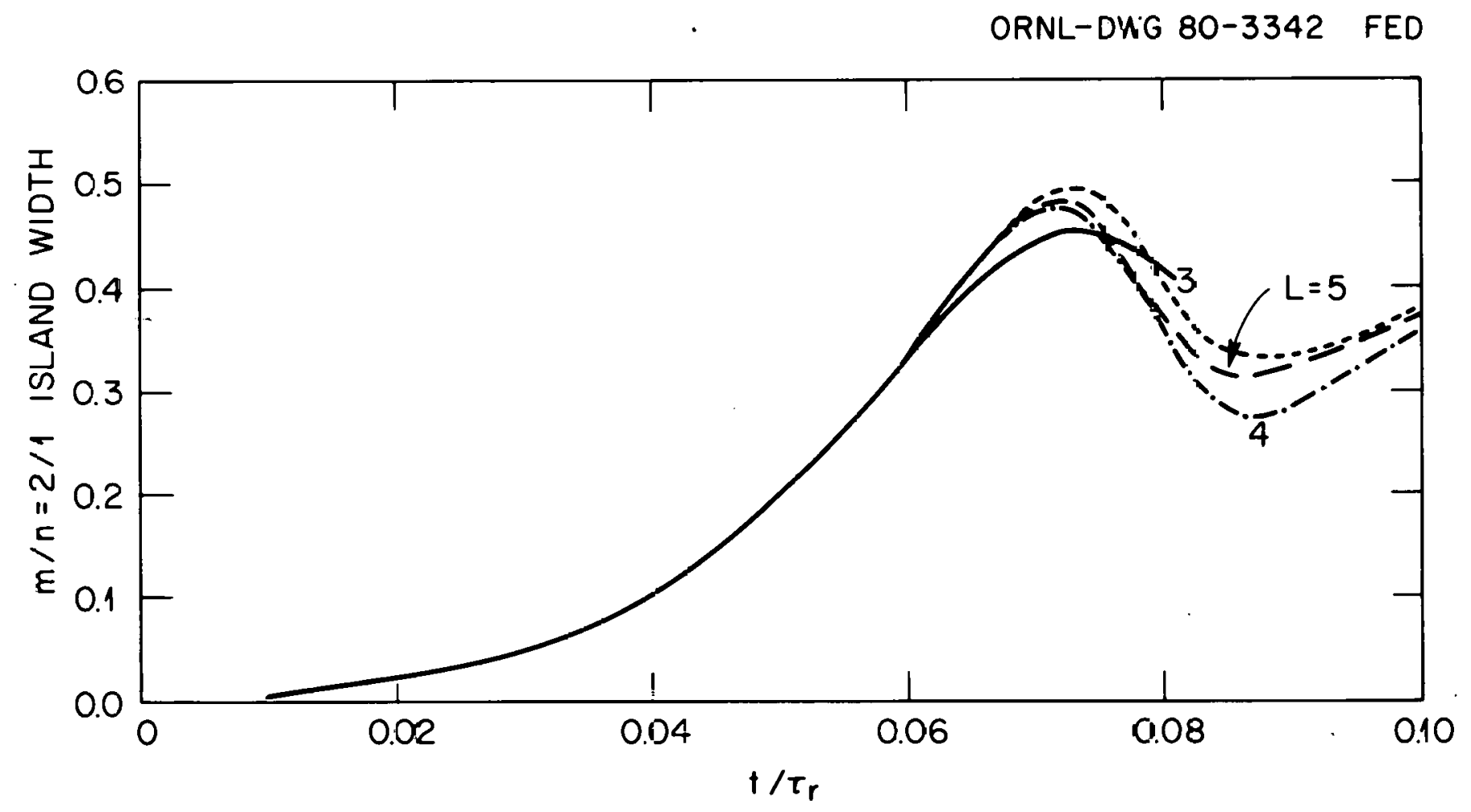

Fig. 4. 


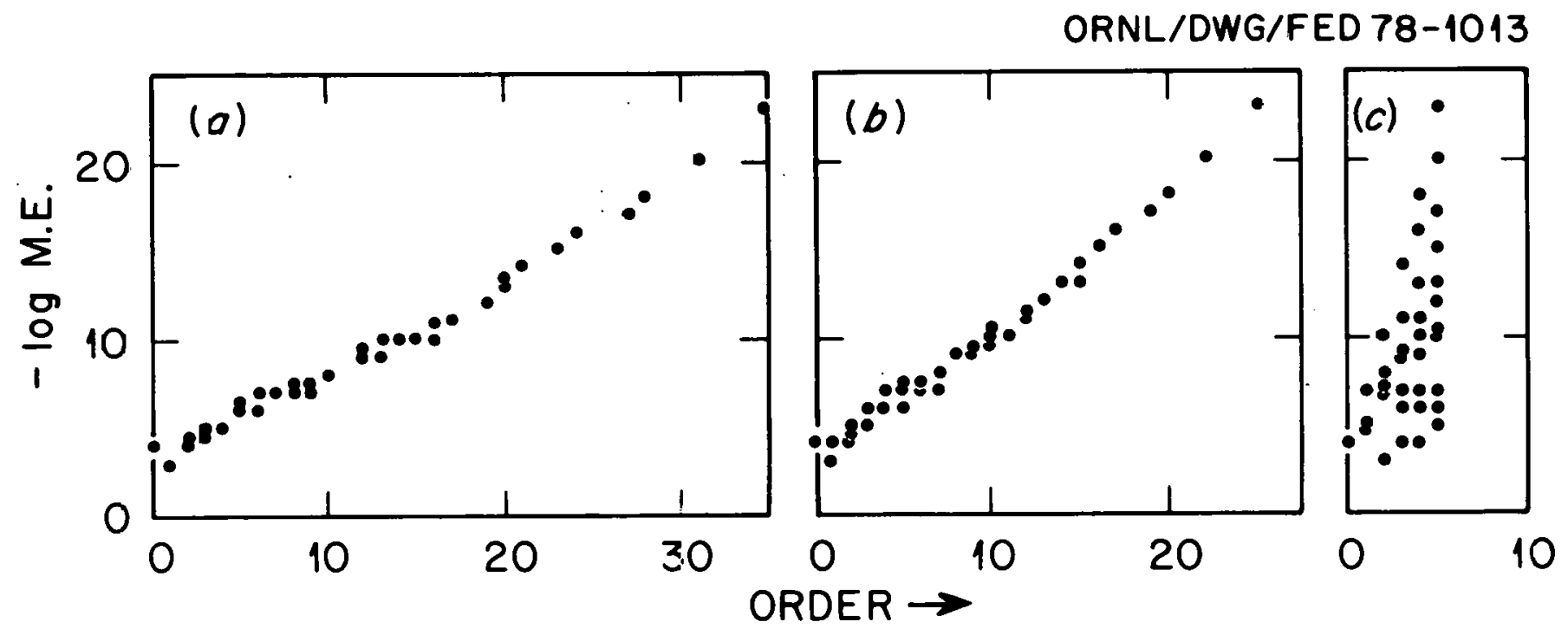

Fig. 5. 


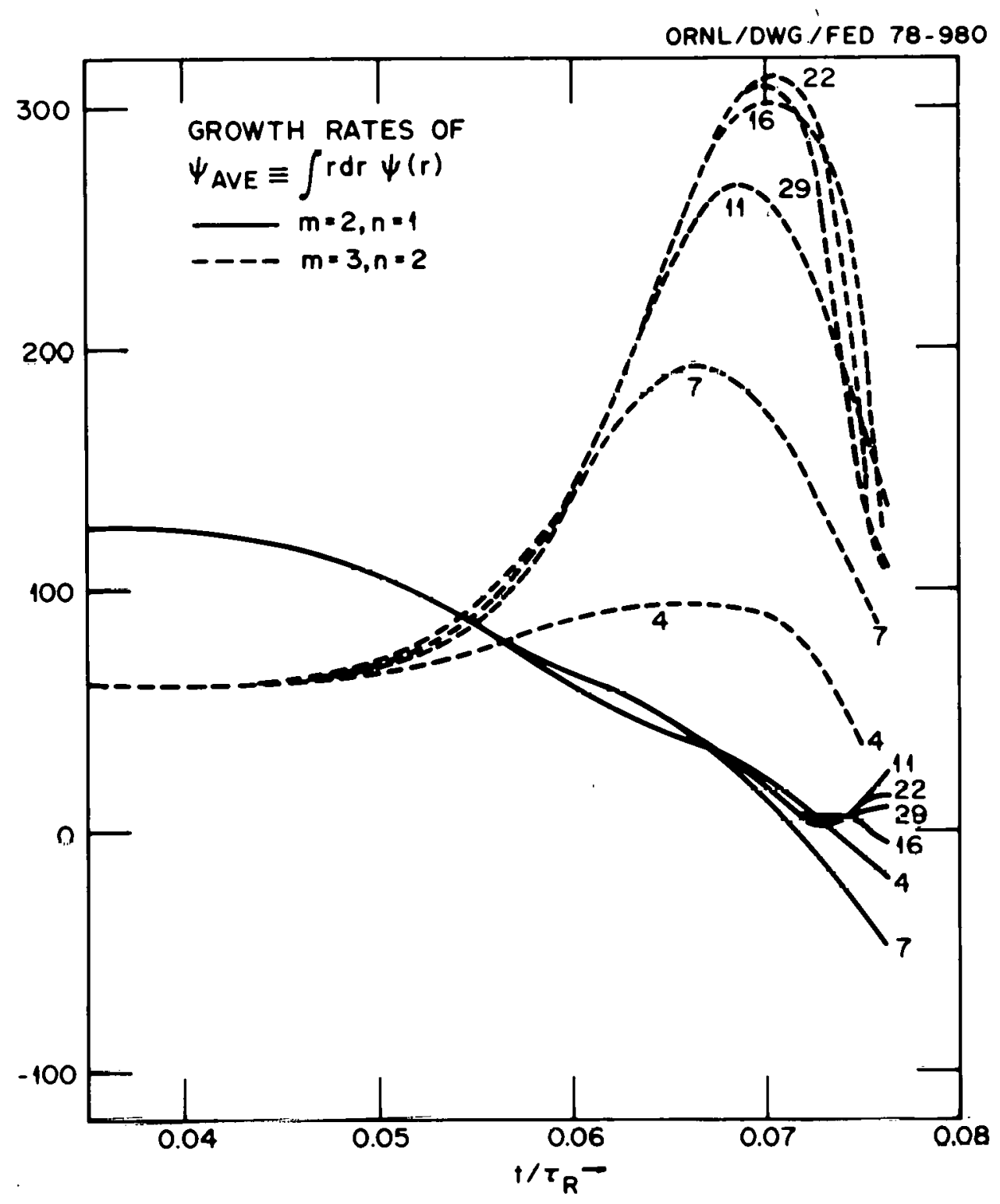

Fig. 6 . 


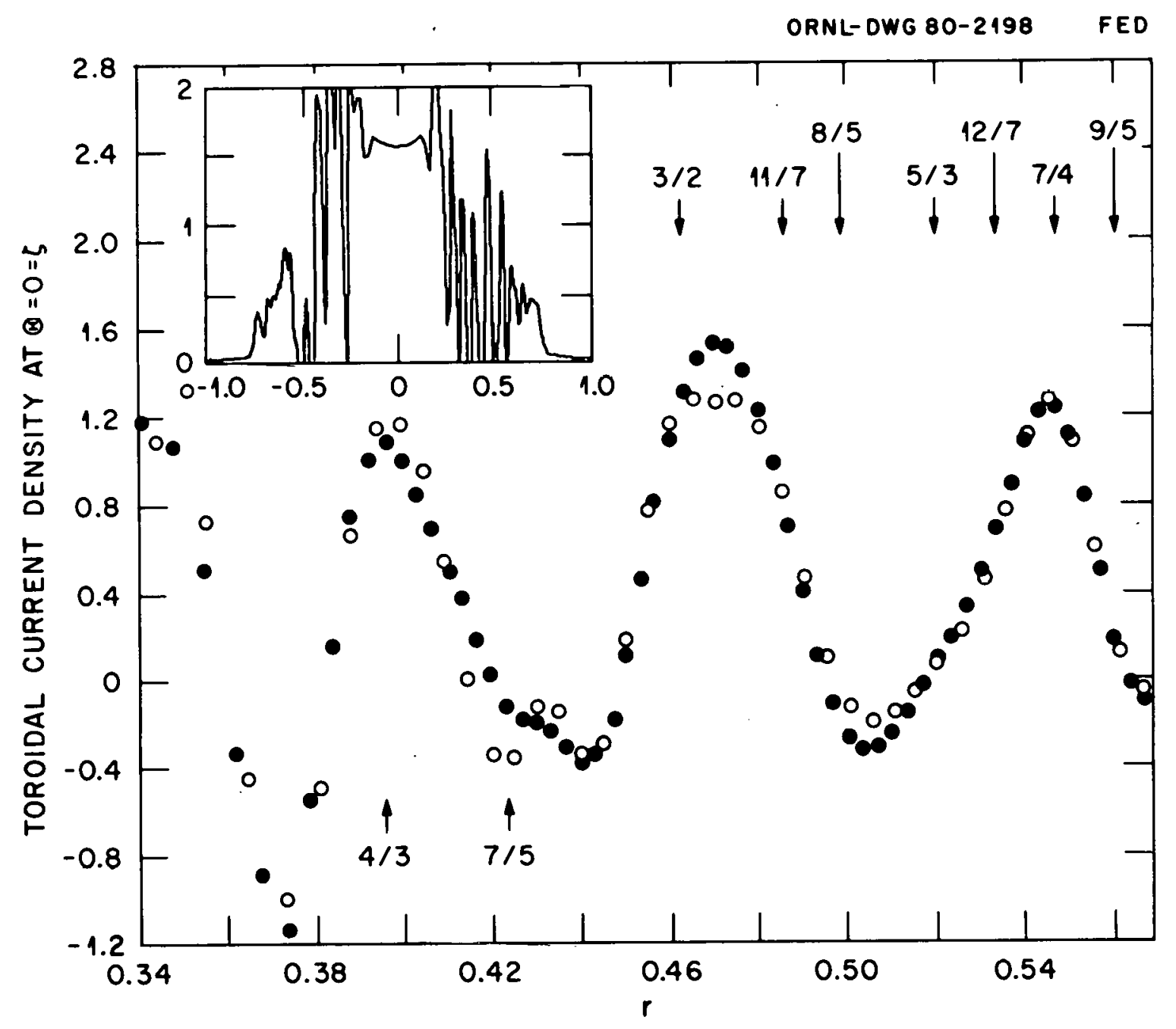

Fig. 7. 


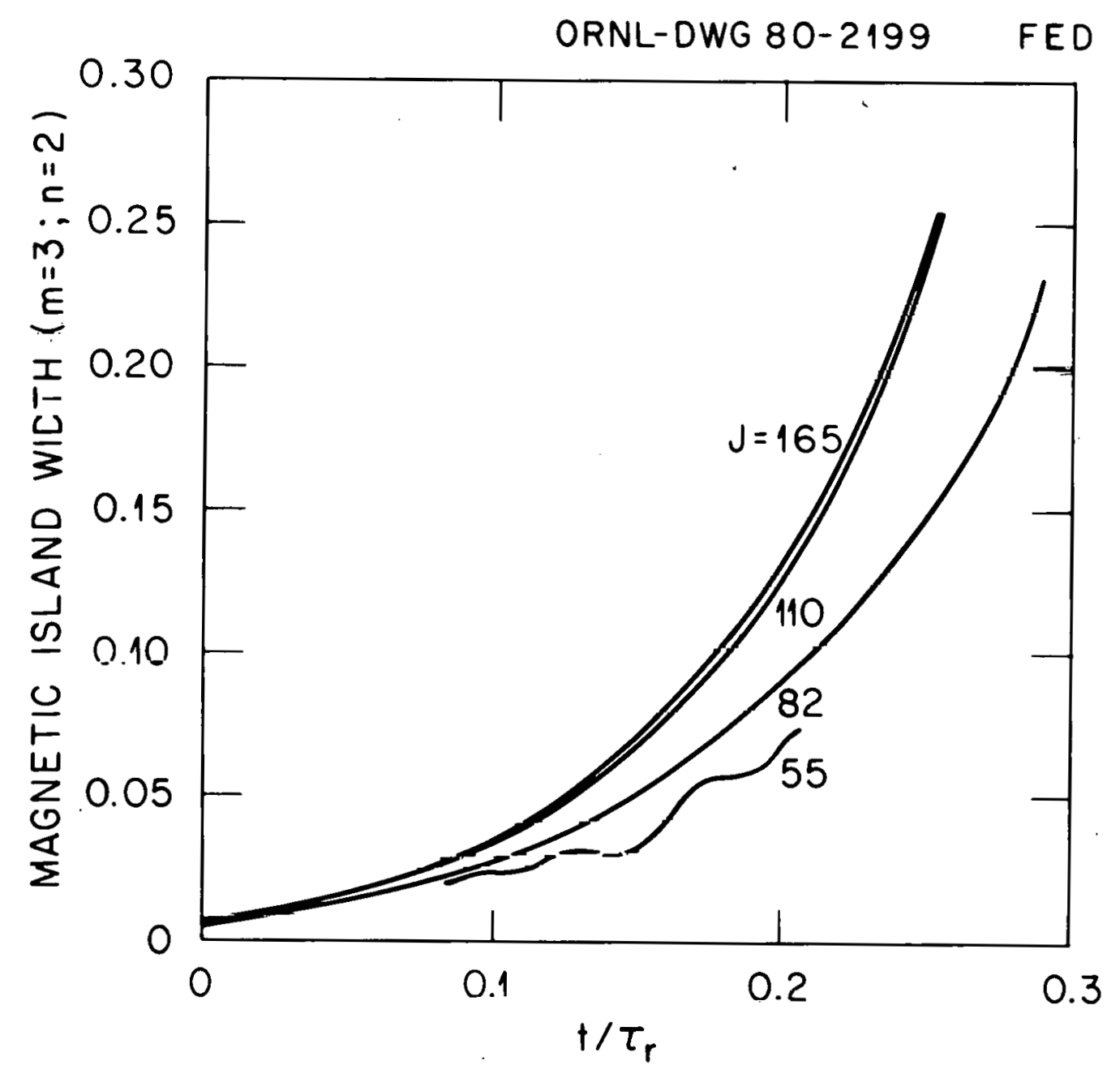

Fig. 8. 
ORNL/TM-7132

Dist. Category UC-20 $\mathrm{g}$

INTERNAL DISTRIBUTION

1-5. B. Carreras

6. R. A. Dory

7-11. H. R. Hicks

12-16. J. A. Holmes

17-21. D. K. Lee

22. L. W. Owen

23. J. Sheffield

24. W. I. van $R 1 j$

25-26. Laboratory Records Dept.
27. Laboratory Records, ORNL-RC

28. Document Reference Section

29-30. Central Research Library

31. Fusion Energy Division Library

32. Fusion Energy Division Reports office

33. ORNL Patent Office

EXTERNAL DISTRIBUTION

34. D. J. Anthony, Manager, Advanced Energy Systems, Energy Systems Program Department, Building 23, Room 290, General Electric Company, Schenectady, NY 12345

35. D. Biskamp, Max-Planck Institut fur Plasma Physik, D-8046 Garching bei Munchen, Federal Republic of Germany

36. J. Brackbill, Courant Institute of Mathematics and Science, New York University, 251 Mercer Street, New York, NY 10012

37. E. P. Caramann, Los Alamos Scientific Laboratory, P.0. Box 1663, Los Alamos, NM 87544

38. R. S. Christian, Purdue University, Lafayette, IN 47907

39. B. V. Chirikov, Institute of Nuclear Physics, 630090 Novosibirsk, U.S.S.R.

40. S. O. Dean, Director, Fusion Energy Development, Science Applications, Inc., 2 Professional Drive, Suite 249, Gaithersburg, MD 20760

41. J. Ford, Georgld Iustitutc of Technn1ngy, 225 North Avenue, NW, Atlanta, GA 30332

42. H. K. Forsen, Vice President and General Manager, Laser Enrichment Department, Exxon Nuclear Company, Inc., 777 106th Avenue, NE, Bellevue, WA 98009

43. H. P. Furth, Princeton Plasma Physics Laboratory, P.0. Box 451, Princeton, NJ 08540

44. R. Grimm, Princeton Plasma Physics Lahoratory, P.0. Box 451, Princeton, NJ 08540

45. B. Grossman, Courant Institute of Mathematics and Science, New York University, 251 Mercer Street, New York, NY 10012

46. R. L. Hirsch, General Manager, Petroleum Exploratory Staff, Exxon Research and Engineering Company, P.0. Box 101, Florham Park, NJ 07932

47. J. Killeen, Lawrence Livermore Laboratory, P.0. Box 808, Livermore, CA 94551

48. R. N. Kostoff, Office of Advanced Technology Projects, Office of Energy Research, Department of Energy, Mail Stop 3F-032, Room $3 \mathrm{~F}-043,1 \mathrm{n} n \mathrm{n}$ Independence Avenue, SW, Washington, DC 20585 
49. L. M. Kovirzhnykh, Lebedev Institute of Physics, Academy of Sciences of the U.S.S.R., Leninsky Prospect 53, Moscow, U.S.S.R.

50. G. L. Kulcinski, Department of Nuclear Engineering, 1500 Johnson Drive, University of Wisconsin, Madison, WI 53706

51. G. Laval, Groupe de Physique Theorique, Ecole Polytechnique, 91 Palaiseau, Paris, France

52. Library, Centre de Recherches en Physique des Plasmas, 21 Avenue des Bains, 1007, Lausanne, Switzerland

53. Library, Culham Laboratory, United Kingdom Atomic Energy Authority, Abingdon, Oxon, OX14 3DB, United Kingdom

54. Library, FOM-Institut voor Plasma-Fysica, Rijnhuizen, Jutphaas, Netherlands

55. Library, Institute for Plasma Physics, Nagoya University, Nagoya 4til, Jafan

56. Library, International Centre for Theoretical Physics, Trieste, Italy

57. Library, Laboratorio Gas Ionizzati, Frascati, Italy

58. J. G. Lominadze, Academy of Sciences of the Georgian S.S.R., 8 Dzerzhinski Street, 38004, Tbilisi, U.S.S.R.

59. D. G. McAlees, Exxon Nuclear Company, Inc., 777 106th Avenue, NE, Bellevue, WA 98009

60. J. E. McCune, School of Engineering, Department of Aeronautics and Astronautics, Building 37-391, Massachusetts Institute of Technology, Cambridge, MA 02139

61. A. T. Mense, Subcommittee on Energy Research and Production, B-374, Rayburn House Office Building, Washington, DC 20515

62. C. Mercier, Service de Theorie des Plasmas, Centre d'Etudes Nucleaires, Fontenay-aux-Roses (Seime), France

63. D. Mikkelsen, Princeton Plasma Physics Laboratory, P.0. Box 451, Princetoin, NJ 08540

64. R. L. Miller, General Atomic Company, P.0. Box 81608, San Diego, CA 92138

65. D. Montice11o, Princeton Plasma Physics Laboratory, P.0. Box 451, Princeton, NJ 08540

66. J. Moser, Courant Institute of Mathematics and Science, New York University, 251 Mercer Street, New York, NY 10012

67. M. Murphy, Uffice of Fusion Energy, Office of Energy Research, Mai1 Station 256, Department of Energy, Washington, DC 20545

68. D. B. Nelson, Office of Fusion Energy, Office of Energy Research, Mail Station 256, Department of Energy, Washington, DC 20545

69. K. Nishikawa, Institute for Fusion Theory, Hirnshima Univcrsity, Higuthisendamachi, H1roshima, Japan

70. B. Outten, Jr., Western Metal Products Company, 1300 Weber Street, Orlando, FL 32803

71. R. E. Papsco, Grumman Aerospace Corporation, 101 College Road, Princeton, NJ 08540

72. R. Pellat, Ecole Polytechnique, 91 Palaiseau, Paris, France

73. 0. Petrus, Supervisor, C.T.R. Theory Group, Plasma Physics Laboratory, AL. I. CUZA University, Iasi, Romania, 6600

74. D. Pfirsch, Max-Planck Institut fur Plasma Physik, D-8046 Garching bei Munchen, Federal Republic of Germany 
75. Plasma Physics Group, Department of Engineering Physics, Australian National University, P.0. Box 4, Canberra A.C.T. 2600, Australia

76. Plasma Physics Library, c/o P. Rosenau, Department of Mechanical Engineering, Technion-Israel, Institute of Technology, Haifa, Israe1.

77. D. Post, Princeton Plasma Physics Laboratory, P.0. Box 451, Princeton, NJ 08540

78. D. C. Robinson, Culham Laboratory, Abingdon, Oxon, OX14 3DB, United Kingdom

79. A. Rogister, Institute for Plasma Physics, KFA, Postfach 1913, D-5170, Julich 1, Federal Republic of Germany

80. M. N. Rosenbluth, University of Texas, Institute for Fusion Studies, RLM 11.218, Austin, TX 78712

81. P. Rutherford, Princeton Plasma Physics Laboratory, P.0. Box 451, Princeton, NJ 08540

82. W. Sadowski, Office of Fusion Energy, Office of Energy Research, Mail Station G-256, Department of Energy, Washington, DC 20545

83. D. Schnack, Los Alamos Scientific Laboratory, P.0. Box 1663, Los Alamos, NM 87544

84. V. D. Shafranov, I. V. Kurchatov Institute of Atomic Energy, 46 Ulitsa Kurchatova, P.0. Box 3402, Moscow, U.S.S.R.

85. A. L. Shestakov, Lawrence Livermore Laboratory, P.0. Box 808, Livermore, CA 94550

86. Y. S. Sigov, Institute of Applied Mathematics of the U.S.S.R. Academy of Sciences, Miuskaya, Sq. 4, Moscow A-47, U.S.S.R.

87. W. M. Stacey, Jr., School of Nuclear Engineering, Georgia Institute of Technology, Atlanta, GA 30332

88. H. R. Strauss, University of Texas, 200 2lst Street, W, Austin, TX 78712

89. J. B. Taylor, Culham Laboratory, United Kingdom Atomic Energy Authority, Abingdon, Oxon, OX14 3DB, United Kingdom

90. V. I. Tereshin, Physica1-Technical Institute of the Ukrainian S.S.R. Academy of Sciences, 310108 Kharkov, U.S.S.R.

91. D. Tetreault, Massachusetts Institute of Technology, 77 Massachusett.s Avenue, Boston, MA 02139

92. Thermonuclear Library, Japan Atomic Energy Research Institute, Tokai, Naka, Ibaraki, Japan

93. K. Toi, Institute of Plasma Physics, Nagoya University, Nagoya 464, Japan

94. N. L. Tsintsadze, Institute of Physics, Academy of Sciences of the Georgian S.S.R., Guramishvili 6, Tbilisi, U.S.S.R.

95. E. Turkel, Courant Institute of Mathematics and Science, New York University, 251 Mercer Street, New York, NY 10012

96. F. Verdaguer, Director, Division of Fusion, Junta de Energia Nuclear, Madrid 3, Spain

97. M. Wakatani, Kyoto University, Kyoto, Gokasho, Japan

98. J. A. Wesson, Culham Laboratory, Abingdon, Oxon, OX14 3DB, United Kingdom

99. C-C. Wu, University of California, 5151 State University Drive, Los Angeles, CA 90032 
100. Office of Assistant Manager for Energy Research and Development, Department of Energy, Oak Ridge Operations Office, Oak Ridge, TN 37830

101-276. Given distribution as shown in TID-4500, Magnetic Fusion Energy (Distribution Category UC-20 g, Theoretical Plasma Physics) 